\title{
Operational performance benchmarking for commercial buildings by using text analytics on work order logs and tenant survey data
}

by

\author{
Saptak Dutta, B.Eng
}

A thesis submitted to the

Faculty of Graduate and Postdoctoral Affairs

in partial fulfillment of the requirements for the degree of

Master of Applied Science in Environmental Engineering

Ottawa-Carleton Institute for Environmental Engineering

Department of Civil and Environmental Engineering

Carleton University

Ottawa, Ontario

March, 2020

(C)Copyright

Saptak Dutta, 2020 
The undersigned hereby recommends to the

Faculty of Graduate and Postdoctoral Affairs acceptance of the thesis

\title{
Operational performance benchmarking for commercial buildings by using text analytics on work order logs and tenant survey data
}

\author{
submitted by Saptak Dutta, B.Eng \\ in partial fulfillment of the requirements for the degree of \\ Master of Applied Science in Environmental Engineering
}

Dr. Burak Gunay, Thesis Supervisor

Dr. Scott Bucking, Thesis Supervisor

Dr. William O'Brien

Dr. Cynthia Cruickshank

Dr. Beatriz Martin-Perez

Professor Yasser Hassan, Chair, Department of Civil and Environmental Engineering

Ottawa-Carleton Institute for Environmental Engineering

Department of Civil and Environmental Engineering

Carleton University

March, 2020 


\section{Abstract}

Operational performance metrics are necessary to detect anomalous floors, equipment, and work-order categories in large commercial buildings. To this end, this thesis presents a method to extract operational performance metrics from computerized maintenance management systems (CMMS) and text-based tenant surveys. The method was demonstrated by using work-order logs and text-based tenant survey data gathered from four large commercial buildings in Ottawa, Canada. The analysis of CMMS data highlights the potential of decision trees, Sankey diagrams and association node networks to effectively visualize anomalies in building complaint patterns. Investigation of the text-based tenant surveys using established text analytics algorithms reveals that classifiers are more accurate for sentiment analysis than lexicon-based methods while both association rule mining and topic modelling algorithms successfully uncover key operational insights. Finally, a software tool mock-up was developed that combines the most impactful elements from previous work for building owners to visualize complaint patterns and maintenance workflows. 


\section{Acknowledgments}

I would like to thank my supervisors Drs. Burak Gunay and Scott Bucking for their patience, knowledge and mentorship. Their encouragement helped me overcome many hurdles I encounteres during my research.

I would like to acknowledge my co-workers at Carleton Building Performance Research Center: Brodie Hobson, Tareq Abuimara, Max St-Jacques, Seungyeong Hong, Adrian Soble, Connor Brackley and Jayson Bursill for their support throughout my studies. They were always willing to collaborate, eager to help and had good feedback and ideas.

I would also like to acknowledge Bentall GreenOak for providing funding as well as data, feedback and support for my research. Specifically, I would like to thank Brandon Malleck - his enthusiasm for collaboration and his passion for improving building performance was inspirational for me.

Finally, I cannot thank my family and friends enough for their unconditional support during the ups and downs during this time. What has been achieved in this thesis wouldn't have been possible without them. 


\section{Preface}

This thesis follows the integrated-article format, consisting two journal articles. The first journal has been published. The second journal is currently under internal review. Readers referring to material from the thesis should cite the current draft. The sections included in this thesis are as follows.

Article 1: Dutta Saptak, Gunay H. Burak, Bucking Scott. 2019. Extracting performance metrics with work-order data. Science and Technology for the Built Environment 1-15, DOI: 10.1080/23744731.2019.1693208.

Article 2: Dutta Saptak, Gunay H. Burak, Bucking Scott. Using data mining techniques to analyze free text tenant surveys. Journal: Science and Technology for the Built Environment.

In the co-authored articles, Saptak Dutta was the principal contributor to the research methodology, data analysis, interpretation and preparing and writing the material presented in the articles, under the supervision of Dr. H. Burak Gunay and Dr. Scott Bucking. 


\section{Table of Contents}

Abstract $\quad$ iii

Acknowledgments $\quad$ iv

Preface $\quad$ v

Table of Contents $\quad$ vi

List of Tables $\quad$ ix

List of Figures $\quad$ X

Nomenclature $\quad$ xiii

1 Introduction 1

1.1 Thesis Overview ........................ 2

1.2 Background on building benchmarking . . . . . . . . . . . . 4

1.3 Research objectives and questions . . . . . . . . . . . . . 7

1.4 Thesis outline . . . . . . . . . . . . . . . . . . 9

2 Extracting performance metrics using CMMS $\quad 11$

2.1 Introduction . . . . . . . . . . . . . . . . . . . 11

2.2 Methodology . . . . . . . . . . . . . . 16

2.2.1 Characteristics of the dataset . . . . . . . . . 16

2.2.2 Analysis approach .................. 18 
2.3 Results and discussion . . . . . . . . . . . . . . . . . 22

2.3.1 Analysis of the complaint $\log$. . . . . . . . . . . . 23

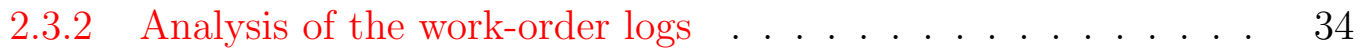

2.3.3 Unresolved issues and future work . . . . . . . . . . . . . . 38

2.4 Conclusions .......................... . . 40

3 Insight extraction using tenant surveys $\quad 43$

3.1 Introduction . . . . . . . . . . . . . . . . . 43

3.2 Literature review . . . . . . . . . . . . . . . . . . . . . . . 44

3.3 Methodology ......................... 48

3.3.1 Characteristics of the dataset . . . . . . . . . . 49

3.3.2 Data preprocessing ................. 51

3.3.3 Text mining algorithms . . . . . . . . . . . . . . 52

3.4 Results. . . . . . . . . . . . . . . . . . . . . . . . 58

3.4.1 Comparison of solicited and unsolicited complaint frequencies $\quad 58$

3.4.2 Text mining algorithms . . . . . . . . . . . . . 59

3.5 Discussion and conclusions . . . . . . . . . . . . . . . 73

4 Development of a graphical interface tool $\quad 77$

4.1 Introduction . . . . . . . . . . . . . . . . . 77

4.2 Methods . . . . . . . . . . . . . . . . . 79

4.2.1 Per category work-order resolution time . . . . . . . . . . 81

4.2.2 Per category work-order intensity . . . . . . . . . . . . 82

4.2.3 Work-order per floor distribution . . . . . . . . . . . 83

4.2.4 Top 10 operator word frequency . . . . . . . . . . . . . 84

4.2.5 Operator term usage patterns . . . . . . . . . . 85

4.3 Future performance metrics and visualization . . . . . . . . . 86 
4.3.1 Temporal distribution of complaints . . . . . . . . . . . 86

4.3.2 Average 12 hour complaint trees . . . . . . . . . . . . 88

4.4 Future application features and development goals . . . . . . . . . . . 89

4.5 Feedback and concluding remarks . . . . . . . . . . . . . . . . . 90

$\begin{array}{lll}5 & \text { Conclusions } & 91\end{array}$

5.1 Summary . . . . . . . . . . . . . . . . . . . . . . 91

5.1.1 Extracting performance metrics with work-order data . . . . . 91

5.1.2 Insight extraction from tenant surveys using text mining . . . 92

5.1.3 A software tool for conducting data analytics . . . . . . . . 93

5.1.4 Envisioned commercial usage ... . . . . . . . . . . 94

5.1.5 Contributions ...................... 95

5.2 Recommendations for future work . . . . . . . . . . . . . . . 95

5.2.1 Improvements on the text analytics methodology . . . . . . . 95

5.2.2 Roadmap for application development . . . . . . . . . . 96

$\begin{array}{ll}\text { List of References } & 98\end{array}$ 


\section{List of Tables}

2.1 An overview of the buildings and the dataset. . . . . . . . 17

2.2 An overview of the ECDF model parameters for the overall complaint patterns in all four buildings. . . . . . . . . . . 25

2.3 A Summary of floors with noted irregularities in the dataset. . . . . . 33

2.4 A Summary of floors with noted irregularities in the dataset. . . . . . 37

3.1 The six topics derived from the thermal and maintenance survey responses using the LDA and NMF algorithms. . . . . . . . . . 63

3.2 Examples of thermal-related tenant survey responses. . . . . . . . . 64

3.3 Examples of maintenance-related tenant survey responses. . . . . . . 65

3.4 Examples of survey responses to thermal issues and their polarity. . . 68

3.5 Examples of survey responses to maintenance issues and their polarity. 68

3.6 Classifiers used and their percent classification accuracy (for negatives and neutrals combined $). \ldots \ldots \ldots \ldots \ldots \ldots$ 


\section{List of Figures}

2.1 An overview of the workflow employed in this chapter. . . . . . . .

2.2 The fraction of unaddressed complaints sorted by category in the buildings using four years worth of data between 2014 and 2017. . . . . .

2.3 An overview of complaint intensity per floor area sorted by category in the buildings. . . . . . . . . . . . . . .

2.4 A breakdown of the temporal variations in the frequency of a) cold complaints b) hot complaints and c) the hot complaints compared to the cold complaints in building one. The mean and standard deviation are annotated with the symbols $\sigma$ and $\mu$, respectively. . . . . . . .

2.5 A breakdown of the temporal variations in the frequency of a) too cold complaints b) too hot complaints between the four studied buildings. The mean and standard deviation are annotated with the symbols $\sigma$ and $\mu$, respectively $\ldots \ldots \ldots \ldots \ldots \ldots$

2.6 Visualizations of a) density distribution of hot complaints during the winter in building four and b) the average temperature on a floor of building four for 24 hours during January 2019 . . . . . . . . . . . .

2.7 Decision trees demonstrating average complaints per 12 hour timespan created for a) hot complaints and b) cold complaints trained using temporal variations e.g. weekdays/weekends, mornings/evenings and outdoor temperatures. The term $T_{\text {out }}\left({ }^{\circ} \mathrm{C}\right)$ stands for the outdoor temperature. . . . . . . . . . . . . . . . 
2.8 Example of a categorical and spatial breakdown of complaints in building two. . . . . . . . . . . . . . . . . . 32

2.9 The top 15 terms used for a) thermal and air quality b) lighting c) maintenance and d) janitorial complaints. . . . . . . . . . . . . .

2.10 Association rules for maintenance related complaints in floor 12 of

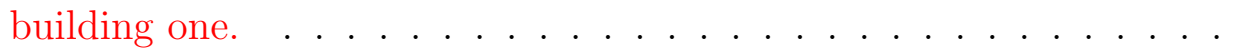

3.1 An overview of the workflow employed in this study. . . . . . . . . . . 49

3.2 A comparison between the per floor breakdown of thermal and maintenance complaints from (a) CMMS and (b) survey responses. . . . .

3.3 Association node graphs built using ARM. The figure contains rules derived for (a) thermal and (b) maintenance-related survey responses. Rules which co-occurred frequently are grouped together and the total number of occurrences for each rule group is denoted by n. . . . . . .

3.4 Average sentiment score and standard deviation of the survey responses for (a) thermal feedback and (b) maintenance feedback on a per floor basis using the TextBlob sentiment analysis library. The number of survey responses per floor is denoted by $n \ldots \ldots$. . . . . .

3.5 Histograms of the response polarity distribution for all survey responses compared with the polarity distribution for survey responses manually verified as negative by the (a) TextBlob and (b) Vader algorithms. . . 67

3.6 An overview of the operations pipeline during binomial classification. $\quad 70$

3.7 A comparison of the confusion matrices obtained from applying the A) Naïve Bayes, B) Logistic Regression C) Support Vector Machine D) Doc2Vec E) Decision Tree F) Random Forest classifiers on the thermal survey responses. . . . . . . . . . . . . . . . . . . . . . 71

4.1 Work-order resolution time benchmarking using survival curves. . . . 81 
4.2 Complaint intensity normalized by floor area using service request counts. 82

4.3 A per floor and per type breakdown of tenant service requests. . . . . 83

4.4 Word frequencies of operator log entries for lighting related service requests. . . . . . . . . . . . . . . . . .

4.5 Word frequencies of operator log entries for lighting related service requests. . . . . . . . . . . . . . . . . .

4.6 Temporal distributions of complaints over a 24-hour period. . . . . . 86

4.7 Decision trees demonstrating the effect of outdoor temporal and spatial factors on average complaint rates over a $12 \mathrm{~h}$ period. . . . . . . . . 


\section{Nomenclature}

\begin{tabular}{ll}
\hline Abbreviation & Full Name \\
\hline \hline CMMS & Computerized Maintenance Management System \\
EUI & Energy Use Intensity \\
LDA & Latent Dirichlet Allocation \\
NMF & Non-Negative Matrix Factorization \\
LEED & Leadership in Energy and Environmental Design \\
HVAC & Heating, Ventilation and Air Conditioning \\
AHU & Air Handling Unit \\
IAQ & Indoor air quality \\
ARM & Association Rule Mining \\
BAS & Building Automation System \\
ANN & Artificial Neural Network \\
RNN & Recurrent Neural Network \\
SVM & Support Vector Machine \\
DTM & Document Term Matrix \\
TF-IDF & Term Frequency-Inverse Document Frequency \\
VAV & Variable Air Volume \\
GUI & Graphical User Interface \\
\hline
\end{tabular}




\section{Chapter 1}

\section{Introduction}

Buildings play a major role on our environmental footprint and economy. While much attention goes into the construction phase of buildings, the operation phase is estimated to account for ten times more embodied energy than the former. It is therefore imperative to develop metrics to benchmark operational performance. Traditional benchmarking techniques depend on simplistic energy-use metrics and demonstrate a high-level overview of building performance, while building audits derive high-resolution operational performance metrics which are time consuming and expensive to conduct. Better methods for characterizing high-resolution metrics are needed to analyze large amounts of data that are collected from commercial buildings today. Low cost and high-resolution benchmarks can be developed using currently underutilized data streams in order to convey detailed performance metrics to building operations and maintenance staff.

A method is proposed to rapidly and automatically extract high-resolution operational metrics from computerized maintenance management systems and text-based tenant surveys. Spatial and temporal factors are used for complaint probability prediction and categorical work order resolution performance benchmarking. In this thesis, text mining techniques such as association rule mining are employed on data 
from four buildings to identify building, system, and component-level work-order taxonomies and common failures. A variety of analytical tools such as topic modelling and sentiment analysis are also utilized for extracting operational insights from yearly tenant surveys. A prototype CMMS analytics software tool is developed based on the text and data analytics methods studied. The shortcomings of the employed workflow are identified; and future research needs are identified regarding the operational data collection, analytics, and visualisation.

\subsection{Thesis Overview}

Holistic performance metrics are necessary to understand how operational resources are used and to detect anomalous zones, floors, equipment, and work-order categories in large commercial and institutional buildings. This thesis is comprised of two studies demonstrating the use of two underutilized data sources to extract operational performance metrics. These two data sources are the computerized maintenance

management systems (CMMS) and the text based tenant surveys. Traditionally CMMS and free text survey data have been overlooked in the field of building performance benchmarking as they are voluminous and unstructured in raw format. Additionally, there is a lack of established tools available which can be used to carry out or streamline the analysis of these data sources. A methodology is developed for conducting text analytics on CMMS and its applicability is demonstrated through a case study in which four years' worth of data from four large commercial buildings are used. Association rule mining technique is employed to identify building, system, and component-level recurring work-order taxonomies and common failure modes. The results highlight the potential of kernel density functions, decision trees, Sankey diagrams, survival curves and stacked line plots to effectively visualize the temporal, 
spatial, and categorical anomalies in the complaint patterns. It is identified that often only a few floors and complaint types account for most of the complaints in a building. For example, in the case study relating to building one, floors three and twelve averaged $89 \%$ and $79 \%$ more complaints than the rest of the building respectively. The analysis of operator comments reveal that the most frequent lighting-related complaints are resolved by replacing ballasts and lights, and the thermal and air quality complaints are addressed by adjusting the temperature setpoints, airflow rates, and fan operation schedules.

The second part of this thesis introduces a method to employ text analytics on tenant surveys. The facility managers of large commercial and institutional buildings periodically collect text-based survey data from their tenants. While these large and amorphous datasets contain valuable information to benchmark operational performance and identify anomalies, it is time and resource intensive to hire employees to read and analyze the datasets and extract insightful information from them. A natural language processing methodology to extract operational insights from text based tenant surveys is created. The effectiveness of this methodology is demonstrated by gaining operational insights from tenant feedback gathered using survey data from a large office building in Ottawa, Canada. Different algorithms for sentiment analysis, association rule mining, and topic modelling are employed in the analysis to consolidate the textual data into common thermal and maintenance complaint categories. The accuracy of different text analytics algorithms is compared; and their appropriateness to analyze tenant survey responses is discussed. Patterns of unsolicited tenant work order requests are contrasted to those observed in the survey responses. The results indicate that the floors that frequently submit work order requests are also likely to submit a large number of negative survey responses. Both work-order and service request data collected in modern buildings represent an 
opportunity to benchmark operational performance and evaluate the effectiveness of operational workflows. However, most CMMS software tools do not go beyond scheduling of work-orders and coordination of trades involved in addressing them, and offer little, if any, insights about the flow of operational resources by time, location, and category. Analytics on CMMS data require custom scripting in programming languages such as $\mathrm{R}$ or Python which are only useable by a small number of specialists. To this end, based on our previous research to extract operational performance metrics, we developed a functional software tool mock-up. The tool is intended to conduct text analytics on CMMS data to extract and visualize operational performance metrics. The software tool is developed by using the shiny package in $\mathrm{R}$ and its functionalities are demonstrated by using the archived CMMS data of four large commercial buildings. Based on the feedback received from the operators of these buildings, a plan for future work is developed. The research presents an opportunity for building owners to implement energy saving measures using existing infrastructure, as well as gain a better understanding of patterns in complaints and maintenance workflows.

\subsection{Background on building benchmarking}

In recent discussions regarding areas of energy saving potential, buildings have come to the forefront of researchers' attention. It is estimated that in several developed nations, the operation of buildings accounts for between 20 and 30 percent of total energy usage [1]. Given such significant contributions to overall energy use, there exists the potential for large energy savings that remain undiscovered in the process of operation and maintaining these buildings. It has previously been estimated that 
up to 20 percent of energy waste in commercial buildings can be attributed to poorly installed and maintained building equipment and components [2]. Benchmarking and studying the operation and failure cycles of building systems and equipment therefore becomes critical to their successful and efficient operation. The ease of use of powerful analytical libraries using common languages like $\mathrm{R}$ and Python allow researchers to efficiently analyze and draw conclusions from large and amorphous data sources such as CMMSs and text-based tenant surveys.

Operational performance benchmarking is critical to the successful management of buildings. However, traditional methods of high-resolution operational performance validation at a component and floor level involve commissioning and can be time consuming to carry out, expensive and difficult to continuously implement. Simplistic methods on the other hand such as the energy usage normalized by floor area (i.e., energy use intensity) may not allow for a high resolution analysis of components within a building system (e.g., thermostats, pumps, fans etc.). In an ideal scenario, there would be continuous commissioning of building components but in most commercial settings this is far too cost intensive to be practical for most building managers. It is also invasive for a tenant if a high resolution (per week, per month etc.) commissioning routine was implemented for all the sensors and subcomponents (thermostats, radiators, valves etc.). On the other hand, large and amorphous data sources such as operator work-order descriptions or occupants' text-based responses to tenant surveys contain invaluable information such as occupant complaint patterns, common operator workflows, problems recurring periodically on certain days of the week, times of the day, seasons, etc. Data from CMMSs can be useful for identifying common faults, operator workflows and component failure frequencies, but they are voluminous, unstructured and difficult to interpret without knowledge of complaint data cataloguing insights [34]. Free text surveys are usually collected 
from tenants on a yearly basis and serve as another critical source of occupant feedback on building/floor level systems and maintenance and building operations routines. However, they are unstructured as a data source and take impractical amounts of time and labour to analyze through traditional manual interpretation methods. Better tools must be developed that are capable of extracting insights from these often-underutilized data streams.

To effectively develop a methodology for analysis of CMMS and text-based surveys, the research is framed into two main segments (1) analysis of CMMS work orders, and (2) analysis of free text tenant surveys.

In the first phase, data from the CMMS of four commercial buildings were extracted, cleaned and analyzed using various techniques in order to generate temporal, spatial and zone level benchmarks. Complaint resolution times and frequencies for four buildings were benchmarked on a per category basis (thermal, lighting, janitorial and maintenance) and complaint probabilities were developed using environmental (temperature) and temporal (day, season) data. Zone level frequencies of complaints were visualized using Sankey diagrams and the breakdown of complaint category patterns on a floor level were studied. Operator workflows and frequent component failures were extracted from log entries by using the association rule mining method. Despite obtaining detailed metrics from the CMMS, the insights were limited to categories which CMMS operators had classified while entering in a complaint. There was a lack of direct and detailed tenant feedback such as the insights obtained from the direct analysis of text-based tenant survey entries.

The second phase of the study, therefore, was based around designing a series of analytical methods which can be incorporated into a workflow for analyzing free-text surveys. Multiple algorithms were used in order to estimate the polarity 
(happiness/unhappiness) of a tenants survey response. Both supervised (classifier based) and lexicon-based techniques were applied to the dataset and their accuracy in predicting the polarity of survey entries was compared. The association rule mining method was used to find frequent co-occurrences of terms used by tenants within the dataset. Finally, two different topic modelling algorithms (LDA and NMF) were applied to the dataset in order to extract the underlying subtopics present within the two main categories of survey response entries: thermal survey responses and maintenance survey responses.

\subsection{Research objectives and questions}

The motivation of this thesis is to develop a method for continuous analysis of buildings systems, components and operator workflows using data driven methods. The method is based on two traditionally underutilized data sources: CMMSs and textbased tenant surveys. Extracting system and component level data is essential for identifying anomalies within systems that can often go unnoticed when using building level benchmarks such as EUI (energy use intensity). The overall research questions that the study aims to answer are:

- Can detailed building and floor level benchmarks for buildings be developed using the data from CMMSs?

- Is it possible to employ text mining techniques to extract actionable insights from text-based survey data?

- Can the CMMS and tenant survey mining and visualization methods be integrated within a prototype software tool? 
The sub-objectives of this research are broken down into the three chapters to address the main research questions as mentioned above. These sub-objectives are listed below on a per chapter basis.

Article 1 (Chapter 2):

1. Understand per building, floor, and zone level breakdown of operational resources.

2. Detect anomalies in the use of operational resources at different spatial resolutions.

3. Interpret anomalies by looking at predictors such as outdoor and indoor temperature, day of week, and time of day.

4. Identify frequent component-level failure patterns.

Article 2 (Chapter 3):

1. Compare the insights gained from unsolicited CMMS complaints to solicited tenant surveys.

2. Develop sentiment analysis techniques in order to discover tenant satisfaction.

3. Employ topic modelling and association rule mining in order to find the most commonly recurring themes within the thermal and maintenance-related survey responses.

Chapter 4:

1. Develop a prototype software tool based on the findings of Chapter 2 and 3.

2. Investigate the most impactful metrics to support operational decision making.

3. Investigate data visualisation strategies to effectively convey CMMS and tenant survey mining results to facility managers. 


\subsection{Thesis outline}

The body of this thesis consists of three main chapters: (1) a methodology for extracting performance metrics using work-order data, (2) a methodology for using data mining techniques to analyze free text surveys, (3) the development of a functional graphical user interface tool for use by non technical users.

Chapter 2: The purpose of this chapter is to develop a method to conduct data analytics on CMMSs and demonstrate its functionality through a case study from four large commercial buildings using analytics techniques such as Sankey diagrams, survival curves and stacked line plots to identify building, system, and component-level recurring work-order taxonomies and common failure modes. Operator comments are analyzed through the association rule mining method in order to reveal the most commonly failing components within the building system. This chapter has been published as Article 1 (Dutta, Gunay, \& Bucking, 2019).

Chapter 3: The focus of this chapter is on developing a methodology to extract operational insights from free text-based tenant surveys. The functionality and accuracy of the workflow is verified using yearly survey data from a large commercial office building. Different algorithms for sentiment analysis are used for deriving tenant polarity, association rule mining is employed for discovering frequently re-occurring word pairs within the dataset, and topic modelling is applied to the data for consolidating the common thermal and maintenance complains into common sub topics which are frequently repeated within the dataset. The insights derived from solicited data (surveys) are also compared with those gained from non-solicited data (CMMS).

Chapter 4: This chapter is based on creating a functional graphical user interface through the R: Shiny package. The application combines the analysis methods 
from the two previous chapters allowing nontechnical users to obtain analytical insights using the aforementioned data and text mining techniques without any prior knowledge of $\mathrm{R}$ or Python. Emphasis is maintained on simplicity, usability and intuitiveness for the user and operator feedback is gathered. Future development goals are discussed and outlined.

Chapter 5: The conclusions obtained in each of the previous chapters are discussed together. The major contributions of the study and the possibilities for future development are outlined and recommendations are made. 


\section{Chapter 2}

\section{Extracting performance metrics using CMMS}

\section{$2.1 \quad$ Introduction}

Operational performance metrics are measurable quantities that indicate progress towards achieving objectives such as energy efficiency, operational cost savings and improvements in indoor environmental quality [3]. At a building level, operational performance metrics act as proxies for building performance and allow operators, and facility and energy managers to continuously monitor and conduct ongoing commissioning in building systems. When developed with archived operations data, these metrics benchmark performance levels for a building, a floor or a type of equipment typical for a time of day, a day of week or a season. Floors, zones, and types of equipment with abnormal performance levels as well as deviations in performance levels over time can be detected by inspecting these metrics. A few examples can be listed as follows: frequency of thermal and air quality, lighting, and maintenance-related

complaints; failure frequency of fans, pumps, chillers, and boilers; average time to address various work-order categories; energy use intensity per floor and occupant.

Although operational performance is a multi-objective construct involving occupant 
comfort and health, tenant satisfaction, workplace productivity, operational cost savings, and energy efficiency, perhaps due to the challenges associated with accessing high-quality operations data, performance monitoring existing buildings often rarely goes beyond crude energy use metrics such as the annual energy use intensity [4]. Related research efforts were directed to develop methods to normalize these simple metrics with historical weather data, which is also known as baseline energy modelling. The baseline energy models can be as simple as degree-day models or three-parameter change point models [5]. They can also be built by using machine learning modelling techniques such as artificial neural networks, decision trees, and Bayesian networks $[6-8]$.

Aside from metrics for energy performance, there are efforts to monitor and benchmark occupant comfort and health, tenant satisfaction, and workplace productivity in existing buildings. The traditional mechanism to benchmark occupant satisfaction with the indoor environment is to conduct tenant surveys [9-11]; however, surveys are time consuming and labour-intensive processes, and cannot be conducted frequently. Furthermore, facility managers may not have the necessary means to analyze the survey results. In the reviewed literature, alternative data sources were sought to develop metrics intended to monitor occupant satisfaction. One of these alternative data sources are human resources databases. For example, Newsham et al. carried out data analytics on a large corporate human resources database to extract metrics such as absenteeism, sick days and employee job satisfaction and performance [12, 13]. They compared these occupant satisfaction metrics in 46 buildings - 13 of which were LEED certified; and demonstrated that manager assessed job performance was slightly greater in the LEED certified buildings than the rest. Despite these case studies demonstrating the use of human resources databases to extract metrics for occupant satisfaction, due to privacy and security concerns it may be difficult for 
facility managers to gain access to these datasets. Two emerging methods to collect data for indicators of comfort are monitoring user interactions with control interfaces (e.g., thermostat keypress, light switch) and soliciting feedback through mobile devices (wearables, phones). For example, Sood et al. presents a method to crowdsource occupant comfort feedback through a smartwatch application [14]. Similarly, based on the hypothesis that occupants tend to interact with their thermostats only when they are thermally dissatisfied, Gunay et al. [15] used the frequency of interactions with thermostats as a metric to assess the user satisfaction with the indoor climate.

O'Brien et al. [16] argues that a shortcoming of performance metrics is the common disregard of building occupancy. For example, despite consuming less energy per person, high occupancy buildings may have greater energy use intensities than their low occupancy counterparts as they deliver building services to a larger number of occupants. In fact, O'Brien et al. [16] compared the use of energy use intensity as a performance metric in calculating a car's fuel efficiency by normalizing its fuel consumption with its empty mass rather than the distance it travels. Recent advances in occupant sensing technologies have enabled the development of occupant-centric building performance metrics. For example, O'Brien et al. [16] put forward several occupant-centric energy use metrics such as the lighting utilization ratio which is the ratio of electric light use duration to the occupied time. Through a simulation-based investigation, Ouf et al. [17] demonstrated that traditional energy performance metrics undermine the value of technologies that improve the adaptability of a building to partial occupancy levels. Demand controlled ventilation and highly granular zoning of electric lighting are two examples of these technologies. Another shortcoming of common performance metrics is that they often consider only a single aspect of performance, e.g. energy performance, occupant comfort. As stated by Hammond 
et al. [18], each metric provides only a narrow perspective of a facility. An energyefficient building may not be perceived as comfortable by the occupants. It may also suffer from frequent building system failures, and thus fall short from the perspective of maintenance. If performance metrics are intended to guide better operational decisions, metrics that measure progress in operation and maintenance holistically are needed.

A source of data that has begun to offer insights into occupant satisfaction and overall operational performance is CMMSs. Occupant complaints (e.g., when a room is too hot) and work-orders issued to address these complaints are often logged within CMMS databases. A technician is then assigned to address the problem - and subsequently enters a brief technical description in the CMMS (e.g., the variable air volume (VAV) terminal unit's damper serving a room is stuck closed). Thus, the complaint and work-order logs within a CMMS contain valuable information to benchmark the overall operational performance. A few examples for the metrics that can be extracted from a CMMS are HVAC-related emergency work-order frequencies, labour time associated with work-orders, lighting-related complaint frequencies, and thermal and air quality complaint frequencies. However, the data in CMMSs are often large and unstructured; and thus, they do not readily lend themselves to human interpretation without employing text analytics.

Text analytics is a process by which large bodies of textual data are analyzed to derive insights and identify patterns. It is generally comprised of three steps: preprocessing, text mining operations, and postprocessing. Text mining operations are the most significant aspect of text analytics and include techniques such as association rule mining (ARM), clustering, and pattern discovery. Text mining represents an untapped opportunity to extract insights from a CMMS. Goins and Moezzi have 
demonstrated the value of having free text comments integrated within non-linear survey questions regarding comfort distributed among participants [19], as these provide venues for participants to provide their own perspective to the relevant teams studying the survey results. However, textual data tend to be overlooked in the field of building performance as their analysis requires tools which till recently were not widely available [20]. Advancements in the ease of use for languages like R and Python have made text mining an accessible tool to researchers in the building performance field [8]. Such advancements have made it possible to analyze and identify patterns in large datasets with the intention of better understand buildings and their common operational anomalies.

The process of monitoring existing buildings remains an open-ended question. Only a few studies have recognized the potential to extract performance metrics from CMMS data. McArthur et al. used CMMS data to classify building-level work-orders [21]; Federspiel (2000) and Wang et al. analyzed the frequency of hot and cold complaints recorded in a CMMS [22,23], Gunay et al. investigated the heating, ventilation, and air-conditioning (HVAC) related fault-frequency rates by using a university campus' CMMS [8], and Bortolini and Forcada introduced a CMMS text analysis method to gain insights into the frequency and severity of maintenance requests [24]. While advances in text analytics enable the development of methods to systematically extract performance metrics from CMMS data, research using such methods in real buildings remains relatively unexplored. This study presents a text analytics-based method to extract performance metrics from CMMS. These metrics are intended to help facility managers understand how operational resources are used, detect anomalous zones, floors, equipment, and work-order categories, and guide ongoing commissioning in large commercial and institutional buildings. The method is demonstrated using a dataset from four commercial buildings in Ottawa, Canada. The limitations of the 
method are identified, and future work recommendations are developed.

\subsection{Methodology}

This section first presents an overview of the CMMS data used to develop and demonstrate the method to extract operational performance metrics. Subsequently, the method to analyze the CMMS dataset is explained.

\subsubsection{Characteristics of the dataset}

In this study, CMMS data from four commercial buildings in Ottawa, Canada were used to develop and demonstrate the text analytics method to extract operational performance metrics. The buildings have multiple tenants and are all served by one large facilities management company. There is a large variance in the characteristics of each building. Building one is the largest building studied and has 19 floors with a total floor area of $111,738 \mathrm{~m}^{2}$ and 138 tenants. This is in contrast to building two which is the smallest studied building and only has five floors with a total floor area of $5,805 \mathrm{~m}^{2}$ and 12 tenants. Given the large dimensional differences between the datasets, it was expected that the algorithms developed for data extraction would have different parameters to tune for.

The majority of the leased-out space consists of offices, with retail shops on the ground floors of the buildings. The age and tenant count of the buildings are listed

in Table 2.1. Buildings one to three operate on a pneumatic HVAC system (with the exception of certain floors in building one) while building four operates on a direct digital control system. The buildings all have dedicated AHUs with perimeter heating units. Most buildings used fluorescent lamps - a mixture of T5, T8, and T12 lamps. 
Table 2.1: An overview of the buildings and the dataset.

\begin{tabular}{|c|c|c|c|c|}
\hline Building & $\begin{array}{c}\text { Floor } \\
\text { Area, } m^{2}\end{array}$ & $\begin{array}{c}\text { Number of } \\
\text { work- } \\
\text { orders }\end{array}$ & $\begin{array}{c}\text { Number of } \\
\text { Tenants }\end{array}$ & $\begin{array}{c}\text { Building } \\
\text { Age }\end{array}$ \\
\hline One & 111,738 & 24,266 & 138 & 1984 \\
\hline Two & 5,805 & 1,104 & 12 & 2006 \\
\hline Three & 14,694 & 4,148 & 6 & 1992 \\
\hline Four & 10,225 & 1,197 & 16 & 1990 \\
\hline
\end{tabular}

The facility managers hired a third-party CMMS provider in order to catalogue and organize occupant complaints. Tenants have the option to contact the thirdparty CMMS service through a phone call or online. Once a complaint is logged, a work-order is generated to address the issue and in-house (or when needed external) technicians are dispatched to resolve the root-cause of the problem leading to the complaint.

In this study, the data from these four buildings were extracted from the CMMS database. The data in the CMMS were collected in a format that was not readily suitable to conduct analytics. The raw data obtained from the database had 230 columns, representing fields such as the date and time of a complaint, the category of a complaint, operator comments, etc. Upon further analysis and discussion with the database maintenance staff, it was decided that only twelve of the 230 columns were maintained regularly and contained relevant information for this analysis. Of the twelve columns, three were text based while the other nine were either in a numerical format (integers) or a date and time format. Building operators recorded complaints into the system by noting a time at which the complaint was opened and writing down when it was resolved. Before analytics could be carried out, the entries where operators may have forgotten to put in a completed date and time were parsed out. The twelve data fields used in this analysis are: (1) building name; 
(2) complaint creation date; (3) complaint completion date; (4) complaint category (e.g., hot/cold complaints); (5) complaint type (e.g., lighting complaints); (6) building floor number; (7) suite number; (8) short description; (9) detailed description; (10) technician comments; (11) work-order priority; and (12) tenant name.

The ideal scenario for this data would be CMMS records without any entry errors

(e.g., un entered complaint completion dates/creation dates, time stamps which are reasonable, completed technician comment fields etc). However, as with all datasets a fraction of the entries were unsuitable for analysis and were excluded from the study. A majority of these were fields where the complaint creation/completion dates were not properly entered.

\subsubsection{Analysis approach}

Complaint creation and completion dates were read as strings by $\mathrm{R}$ and converted to date numbers. By using $\mathrm{R}$ packages anytime and lubridate, the response time to a complaint was calculated as the number of hours from beginning to the completion of the request. Overall, five different metrics were computed by using the response time data: overall response time, response time to lighting complaints, response time to thermal and air quality complaints, response time to janitorial complaints (e.g., washing carpets, cleaning washrooms, replacing soap) and response time to maintenance complaints. The complement of the empirical cumulative distribution was computed to build survival models predicting the likelihood of observing a period without any complaints. This process was first carried out for all complaints, and then individually for the four complaint types (i.e., lighting, thermal and air quality, janitorial and maintenance). Further, the complaints were separated by date and complaint type (lighting, thermal and air quality, janitorial and maintenance). The sum of all complaint types between the years 2014 and 2017 was calculated 
and divided by the floor area of the building in order to determine the complaint intensity normalized by the floor area. The overall complaint intensity per floor area was derived by adding the complaint intensities of different categories within an entire year. By using the $\mathrm{R}$ package ggplot2, this is demonstrated over a four-year span using a stacked line chart to show trends in the data.

By using the R package dplyr, thermal (hot/cold) complaints were extracted from the dataset in order to study the effects of temporal changes on complaint frequency. The temporal categories used to divide the complaints were as follows: weekdays (Monday to Friday) and weekends (Saturday and Sunday), Summer (May to August), Winter (November to February), Shoulder (March, April, September, and October) seasons, and mornings (before $12 \mathrm{pm}$ ) and afternoons (after $12 \mathrm{pm}$ ). Probability distribution functions were then calculated for each categorical split and the mean and standard deviation were calculated in order to ascertain whether the differences observed in the temporal effects had empirical significance. Anomalies found while exploring the thermal and air quality complaints were further investigated by studying the indoor temperature records extracted from the building automation system. The effects of outdoor temperature alongside the previous temporal categories on complaint intensity were studied using decision trees. Daily outdoor temperature data for a period of four years was obtained from a local weather station and appended to the complaints. These categories were used to train two decision trees through a binary recursive partitioning algorithm (using the $\mathrm{R}$ package rpart) for hot complaints and cold complaints, respectively. For verification, multiple trees were built through repeated random sampling from the complete dataset - i.e., cross-validation.

The complaints were grouped in four categories and all complaints not belonging to one of the four categories were grouped in as a new category called "other". Recall 
that these four categories were thermal and air quality, lighting, maintenance, and janitorial. A matrix of the per floor complaints and their categorical breakdowns were used to build a Sankey diagram (using the $\mathrm{R}$ package networkD3). Thicker Sankey branches indicate greater numbers of complaints. This visualization is intended to pinpoint problematic areas where there are anomalous complaint rates. Note that the Sankey diagrams included in this study are static diagrams; while interactive Sankey diagrams could provide further details.

Text analytics was used on the three text-based data fields containing uncategorized data. The R packages $t m$ and SnowballC were used to remove punctuation marks, stop words, suffixes and prefixes. Numbers were removed from the dataset and all letters were converted to lowercase. A document term matrix is then created from the processed data for further analysis. The rows of the document term matrix contain the work-order number and the columns contain all the words used within the studied document. Words that were very sparse were filtered out and removed. The document term matrix was used to generate a bar graph of the top 15 words used in lighting, thermal and air quality, janitorial and maintenance related work-orders. The ARM method - which is a text mining technique to identify frequent itemsets within a dataset by using the Apriori algorithm was then employed. In this study, we employed ARM to identify word combination patterns that were repeatedly used in describing the work-orders. The most important parameters while dealing with ARM are support, confidence and lift. Support is defined as a measure of the proportion of instances in a dataset that an itemset appears (e.g., fraction of work-orders in which several terms co-exist). A high support value for a rule is an indication that the rule exists among multiple entries in the dataset. The second metric confidence represents the proportion of work-orders with the word $\mathrm{X}$ where the word $\mathrm{Y}$ also exists. A high confidence value indicates the level of confidence that 
an itemset does not exist based on pure coincidence. Hence, it is favourable to find itemsets with high support and confidence levels. The third metric lift is a measure of the probability of a rule appearing together in a dataset divided by the product of the individual probability that each word making the rule appears separately. A lift value greater than one suggests that there is a positive correlation between words $\mathrm{X}$ and $\mathrm{Y}$. A value of one suggests no correlation and a value between zero and one suggests that there exists a negative correlation between the words. The Apriori algorithm was used to generate a list of word coexistences with pre-defined support and confidence thresholds (by using the $\mathrm{R}$ package arules). Association node network graphs were built presenting the top rules highlighting top word combinations in the dataset by support, confidence, and lift. Due to the variation in the size and complexity of the datasets from individual buildings, the support, confidence and sparsity thresholds used by the ARM algorithm were manually tuned for each building. Additionally, words which were unique to the dataset such as the building manager names, and names of janitorial staff were removed through manual inspection. Note that while other facilities may have different CMMS work-order and complaint categories, lighting, thermal and air quality, janitorial, and maintenance were the only categories available in the CMMS data used in this study. Figure 2.1 demonstrates the workflow that was followed 


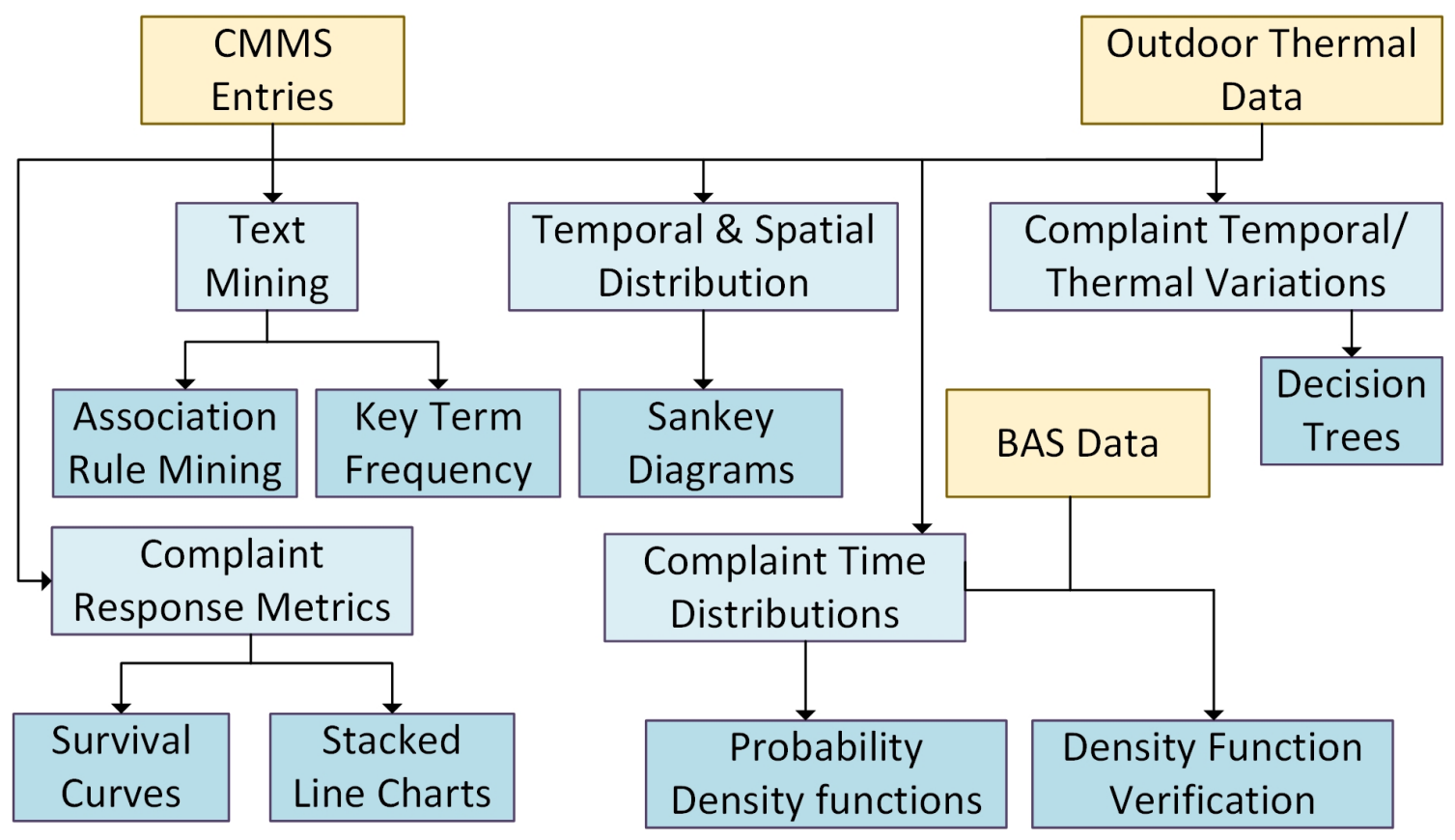

Figure 2.1: An overview of the workflow employed in this chapter.

\subsection{Results and discussion}

In order to derive insights from the data, the extracted complaint logs were analyzed based on their type and temporospatial distribution. Techniques to analyze and visualize the complaints were examined. Seven metrics to monitor complaint patterns and to detect and isolate anomalies were developed. Five of these metrics were derived from the complaint logs: fraction of complaints unaddressed in a given time interval, complaint intensity per floor area, temporal distributions of thermal and air quality complaints, decision tree driven complaint frequencies, and categorical and spatial breakdown of the complaints per area. Two of them were derived from operator descriptions while addressing the work-order requests: frequency of key terms and association node networks. 


\subsubsection{Analysis of the complaint logs}

\section{Fraction of complaints unaddressed in a given time interval}

The survival models shown in Figure 2.1 demonstrate the performance of a building with respect to how quickly complaints are addressed. For example, the thermal and air quality and janitorial complaints in buildings four and two took longer to address compared to the other buildings in the dataset. They had a $75 \%$ and $90 \%$ unaddressed rate at the 10-hour mark compared to all the other buildings having an unaddressed rate of below $50 \%$ for those two parameters within the same time frame. The two buildings underperforming regarding addressing lighting issues were buildings one and four. They had $78 \%$ and $75 \%$ of their respective lighting complaints unaddressed within 15 hours compared to the rest of the buildings having under $65 \%$ of their complaints unaddressed. While building four was an anomaly, lighting performance issues were expected for building one as the building was going through a lighting retrofit and many clients had problems with the newly installed lighting fixtures. The survival curve addressing thermal and air quality complaints in building four showed that $55 \%$ of the complaints went unaddressed within a 10-hour period. This compared unfavourably to the rest of the studied buildings which had less than $40 \%$ of their thermal and air quality complaints unaddressed in the same time span. One would anticipate that the time taken to address a work-order should correlate to its severity. Yet the priority of the work-orders field was not routinely maintained to test this hypothesis. The priority of the work-orders field contained only two options: high and low priority; and most of the work-orders were categorized as high priority despite many of them being basic actions. Future research should investigate the effect of work-order priority on this metric whereby the severity of the work-orders is better structured. Table 2.2 records the model 
parameters of the ECDF functions for the overall complaints category for all four buildings.
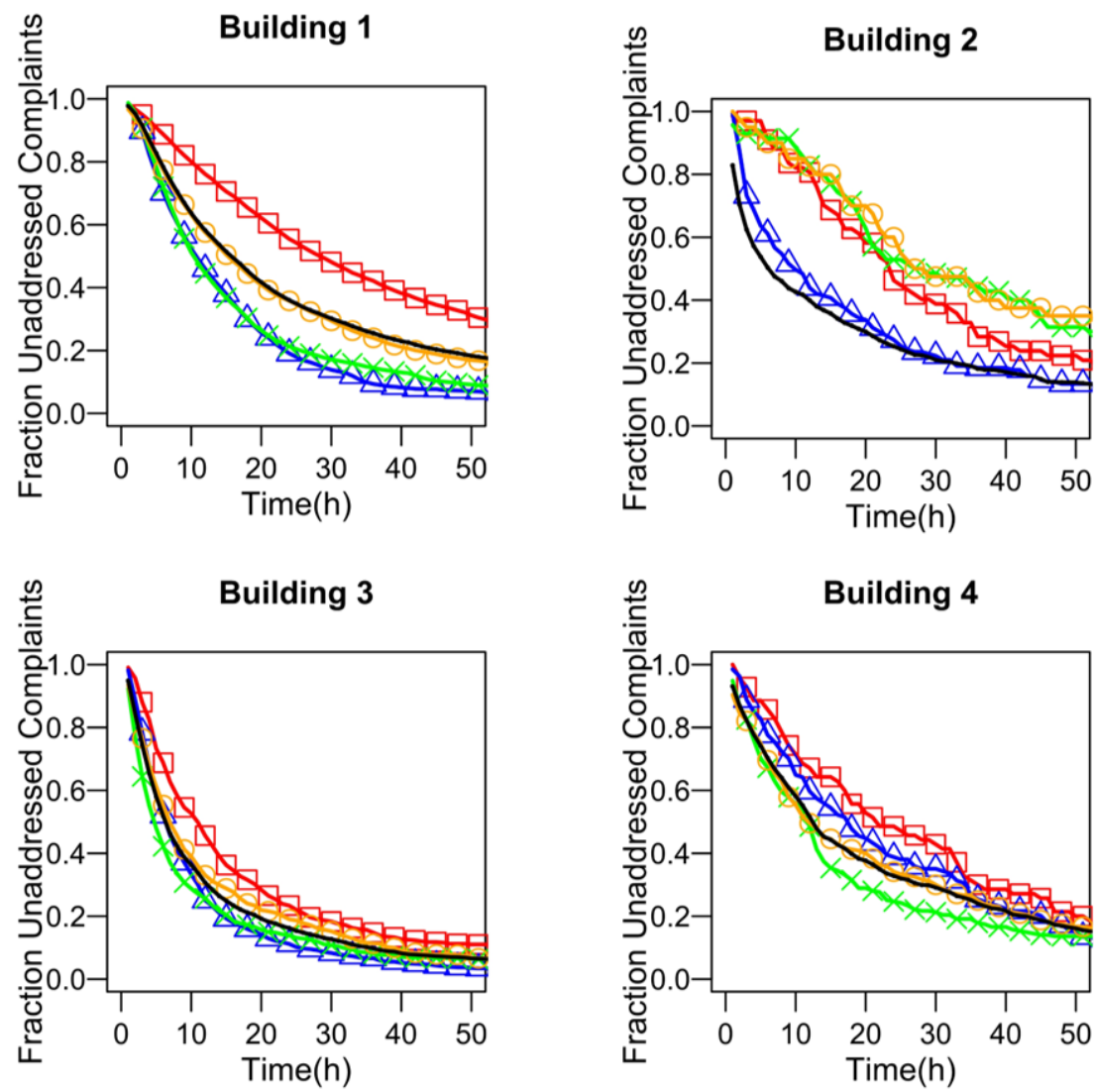

Survival Curve Categories

$\longrightarrow$ Overall

...-.. Lighting

$\triangle$ Temperature

$\cdots \times$ Janitorial

- Maintenance

Figure 2.2: The fraction of unaddressed complaints sorted by category in the buildings using four years worth of data between 2014 and 2017. 


\begin{tabular}{|c|c|c|c|c|c|c|}
\hline Building & Minimum & 1st Quarter & Median & Mean & 3rd Quarter & Maximum \\
\hline 1 & 0.0003 & 1.0126 & 2.3081 & 5.3295 & 5.3158 & 24 \\
\hline 2 & 0.0003 & 0.1883 & 0.7417 & 3.0310 & 2.6103 & 24 \\
\hline 3 & 0.0006 & 0.3581 & 0.7874 & 1.9550 & 1.8871 & 24 \\
\hline 4 & 0.0017 & 0.5150 & 1.2842 & 3.8254 & 3.5918 & 24 \\
\hline
\end{tabular}

Table 2.2: An overview of the ECDF model parameters for the overall complaint patterns in all four buildings.

\section{Complaint intensity per floor area}

Stacked line charts are useful for demonstrating the change in complaint intensity per square meter for a building. As shown in Figure 2.2, interesting observations for building one include an overall decrease in lighting complaints per square meter by around $57 \%$ between 2014 and 2017. In contrast, janitorial complaints increased by around $27 \%$ in that same time frame. The buildings two and four showed clear reductions in the number of complaints per square meter at $71 \%$ and $77 \%$, respectively. The largest reductions in complaint intensity observed for building two were in thermal and air quality and janitorial complaints at $60 \%$ and $56 \%$, respectively. For building three, the most notable reductions were in janitorial and maintenance complaints at a 54\% and 58\% decrease, respectively. 2014 was an outlier for building four as the number of complaints reported was only $24 \%$ of the total 2017 value. The trends in building 1 indicate that facilities management team were successful in improving their workflows between 2014 and 2016. However, in 2018 there was a major renovation which could have led to disruptions in previously established maintenance routines causing the increase in complaint intensity. Further research must be done into the specific causes for the reduction in complaints. 

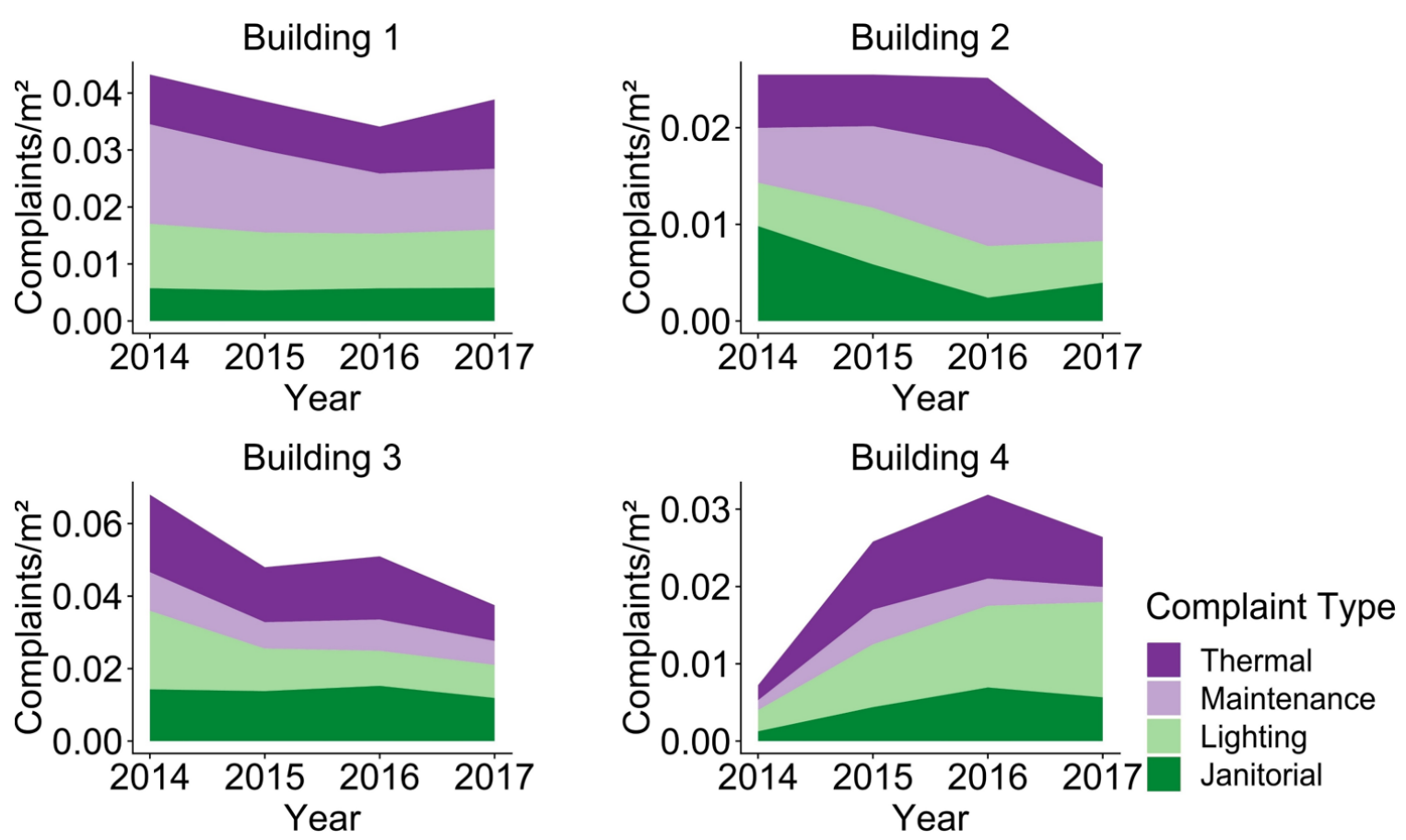

Figure 2.3: An overview of complaint intensity per floor area sorted by category in the buildings.

\section{Temporal distributions of too hot/too cold complaints}

Probability density functions were used to visualize the temporal variations in the frequency at which too hot or too cold complaints were logged in building one. Figure 2.3 shows how the hot and cold complaints vary during the different seasons. The peak complaint probability for all the categories appeared to be around 10 am. The probability density functions for heating, cooling and shoulder seasons shared similar structures for both hot and cold complaints. When the shapes of the density functions for the hot and cold complaints were compared to each other, they appeared distinct. The hot complaints had a secondary peak which may have been caused due to overheating of the space because of solar heat gains. Figure 2.4 presents the probability density functions for the thermal and air quality complaints throughout the year in order to demonstrate the variation in density function patterns between the four studied buildings. Building three appeared to 
have the earliest peak in thermal and air quality complaints (approximately 9 am), indicating that the building may be inadequately conditioned in the mornings as tenants enter the space. Additionally, it has the most prominent secondary peak for hot complaints indicating possible overheating during the afternoon. The mean cold complaint time for building two is at 12:30 pm with a lag of 30-minutes compared to the other buildings. The mean hot complaint time for building one is at $2 \mathrm{pm}$ with a three-hour deviation from the means of the other buildings. Building one is also observed to have the largest standard deviations for the hot and cold complaint categories. The temporal complaint distribution curves can give building staff the ability to spot irregularities in patterns such as secondary complaint peaks and tailor the schedules and setpoints of their HVAC systems accordingly.
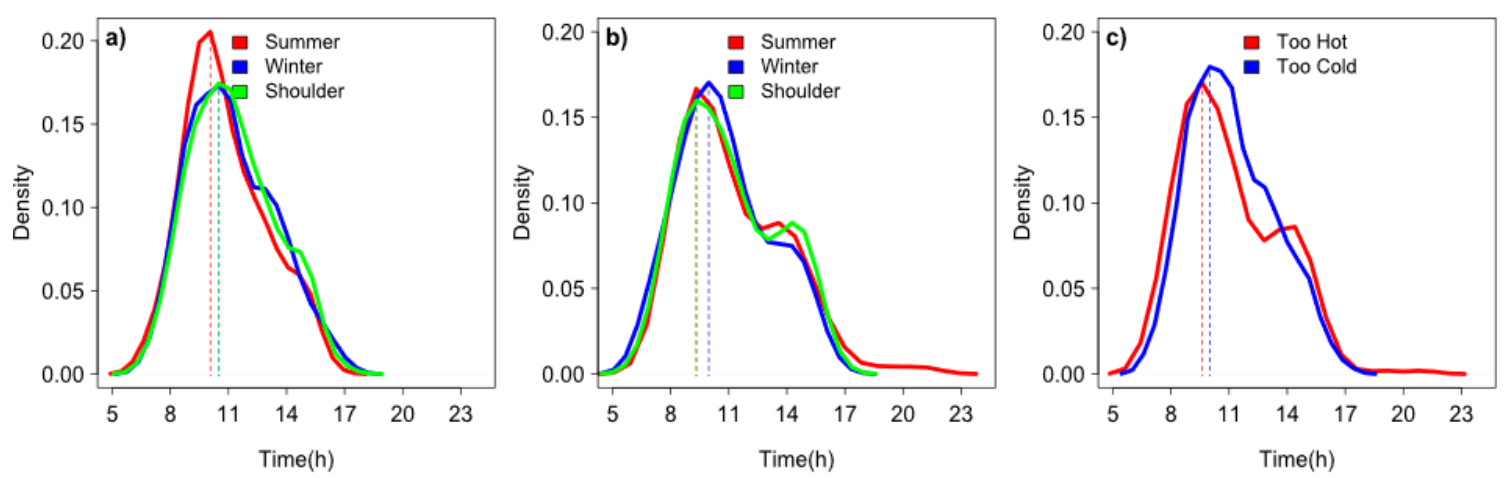

Figure 2.4: A breakdown of the temporal variations in the frequency of a) cold complaints b) hot complaints and c) the hot complaints compared to the cold complaints in building one. The mean and standard deviation are annotated with the symbols $\sigma$ and $\mu$, respectively. 

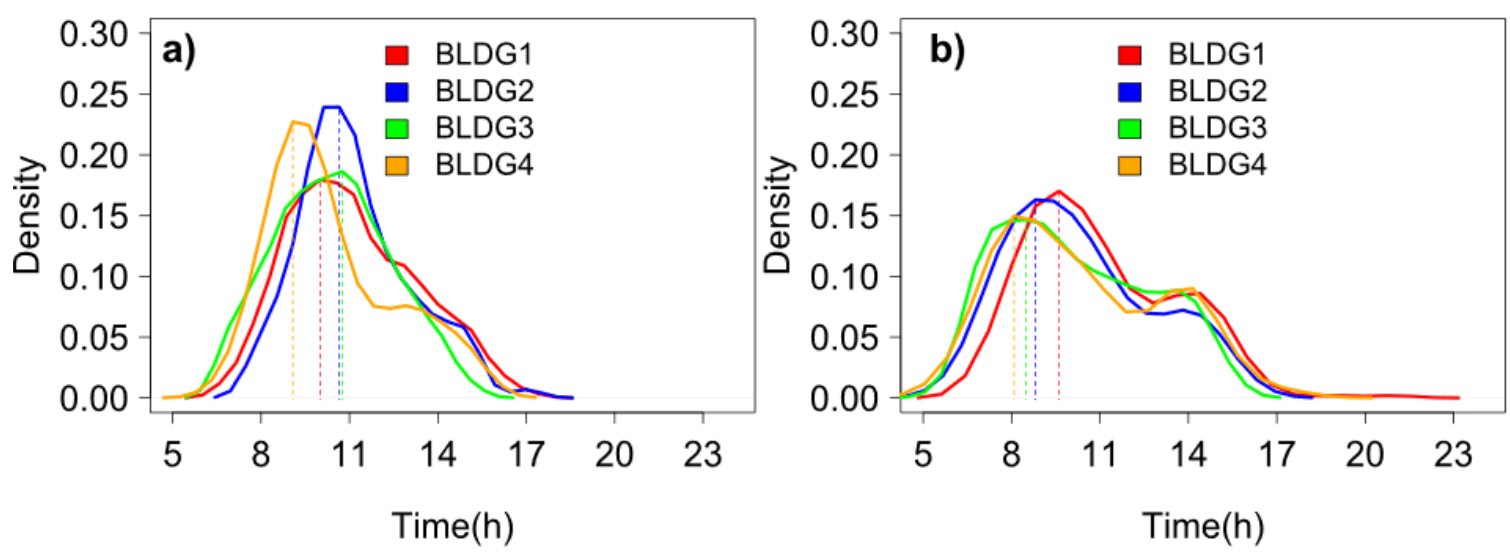

Figure 2.5: A breakdown of the temporal variations in the frequency of a) too cold complaints b) too hot complaints between the four studied buildings. The mean and standard deviation are annotated with the symbols $\sigma$ and $\mu$, respectively

\section{Inspection of the temporal hot complaint distributions}

The anomalies discovered while visualizing the temporal trends found on the probability density functions in building four were further explored by visualizing the trend of the average floor temperature for 24 hours over a period of 31 days in January 2019. Figure 2.5 shows the density distribution alongside the floor temperatures to compare the timing of the secondary peak in complaint frequency with the indoor temperature. It can be observed that the secondary peak coincided in time with the highest average indoor temperature (approximately $22.5^{\circ} \mathrm{C}$ ). This was a possible indication of an overheating issue within the space causing occupant discomfort at $2 \mathrm{pm}$, leading to an uptick in the complaint frequency. The increase in temperature may be attributed to solar heat gains. The timing of the temperature increase (during the afternoon) supports this hypothesis as the increasing solar gains would cause a temperature peak in the space in the afternoon. Ideally, the hypothesis that the space was overheating would be investigated by exploring the zone level temperature data on a per floor basis on multiple floors. Unfortunately, at the time of verification, the inspection was only done on one of the floors because 
temperature data from the rest of the floors were not available. The addition of data collection capabilities to store the data at the zone-level can be useful for future analysis. There is also a possibility of non thermal factors causing an irregularity in the complaint curve. The decrease in complaints at $12: 00 \mathrm{pm}$ could be attributable to tenants leaving the office for lunch, and the secondary peak being lower than the primary peak at the 9 pm- $10 \mathrm{pm}$ time interval could be due to a reduced willingness to complain as most occupans would leave the space in a short time (approximately 3-4 hours). A second source of uncertainty is the lack of occupancy data in these floors. Ideally complaints would be normalized by occupancy to account for days with a lower number of tenants.
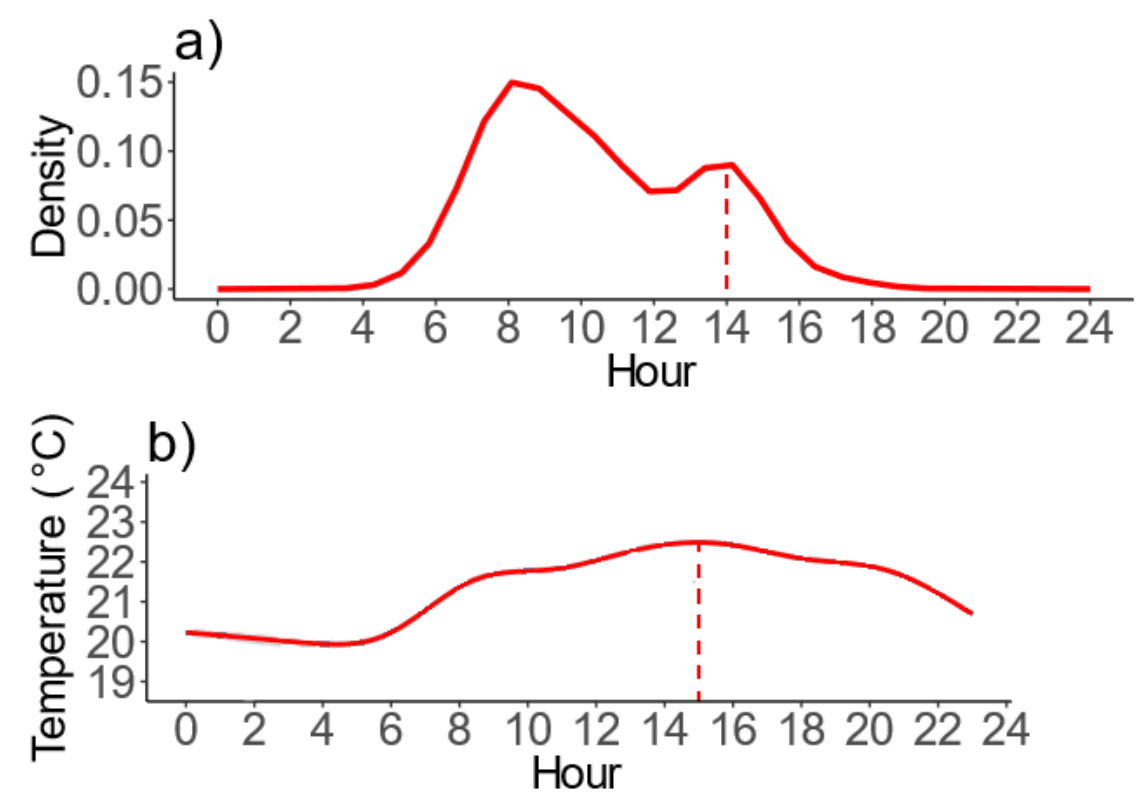

Figure 2.6: Visualizations of a) density distribution of hot complaints during the winter in building four and b) the average temperature on a floor of building four for 24 hours during January 2019 


\section{Decision tree driven complaint frequencies}

Decision trees present the effects of temporospatial factors on the number of hot and cold complaints that are logged in a day. The decision trees shown in Figure 2.6 were constructed using CMMS complaint data from building one. The two decision tree models were developed for hot and cold complaints taking into account four years of complaint logs. The variable $\mathrm{n}$ within each tree refers to the number of 12-hour timesteps in a bin within the dataset. The decision tree consists of nodes (squares) and leaves (circles). The nodes of the tree quantify statistically significant splits within the dataset while the leaves demonstrate the average number of complaints that would occur within a 12-hour timespan. The three most significant splits found for both the hot and cold decision trees were days of the week (weekdays/weekends), outdoor temperature and the time of day (mornings/evenings). Only one hot complaint and three cold complaints were logged during the four-year study period during weekends. The seasonal effect on the number of complaints (winter/summer/fall) was found to be significant only when considering cold complaints. The highest complaint frequency for the cold complaints tree occurred during summer mornings when the outdoor temperature was less than $10^{\circ} \mathrm{C}$, indicating that the space was being over cooled. The two highest complaint frequencies occurred only during the summer months with the tree splitting the winter and shoulder season cold complaints separately. The highest average rate of too hot complaints occurred on weekdays when the outdoor temperature was greater than $22.5^{\circ} \mathrm{C}$. This can be interpreted as building one struggling to provide the adequate cooling on warmer days. It should be noted that the highest average number of complaints were located in bins which only have a small number of time intervals. Ideally the decision trees would be developed on datasets with a larger number of data points for a more representative spread of data. The decision trees 
provide insights regarding conditions associated with high hot/cold complaint rates, which can be used to inform seasonal and daily changes in the operating schedules and setpoints of the building HVAC systems. Considering that building one has a modern BAS, these insights could easily be used in making such operational decisions.

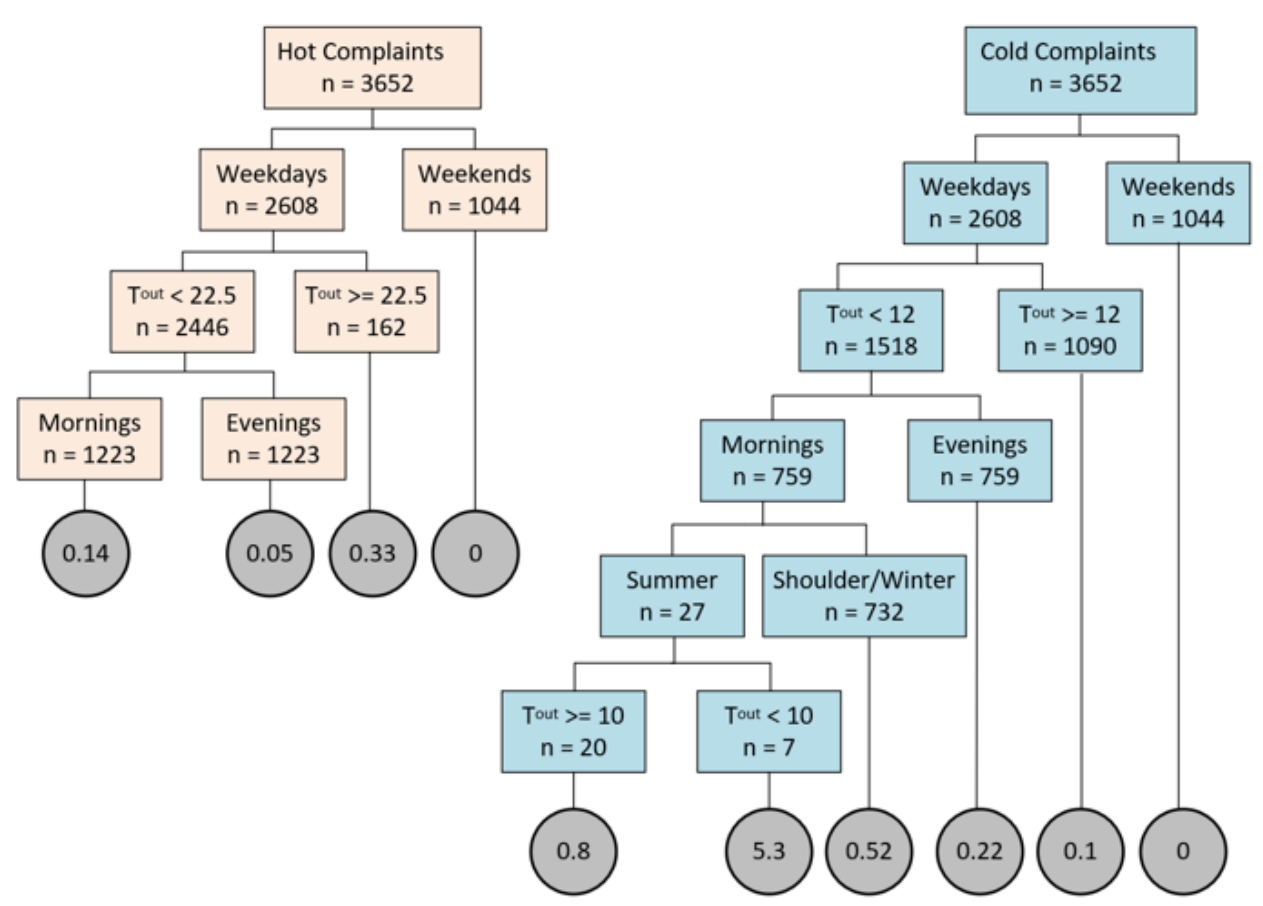

Figure 2.7: Decision trees demonstrating average complaints per 12 hour timespan created for a) hot complaints and b) cold complaints trained using temporal variations e.g. weekdays/weekends, mornings/evenings and outdoor temperatures. The term $T_{\text {out }}\left({ }^{\circ} \mathrm{C}\right)$ stands for the outdoor temperature.

\section{Categorical and spatial breakdown of the complaints per area}

Sankey diagrams provide a visualization for the categorical and spatial breakdown of the complaints. For example, often only a few floors and complaint categories account for a large fraction of the complaints. Presenting a per floor and category breakdown 
of the complaints provides insights into areas where problems are expected and areas where key operational improvements can be made to improve tenant satisfaction. For example, the largest category of complaints originating from the third floor of building two was related to lighting (see Figure 2.7). The most frequent action in response to these lighting-related complaints was changing the ballasts. This is expected as the studied buildings had been retrofitted with new fluorescent lighting fixtures. A common reason for lighting failures is due to the old ballasts being used on new fluorescent lighting fixtures despite being incompatible. The two prevalent issues under the janitorial complaints category were the requests for cleaning carpets and removing the garbage. Having a more frequent maintenance schedule for carpet cleaning and waste management would address these common issues and could have a positive impact on tenant satisfaction and comfort. Table 2.2 lists all floors with irregularities found through the exploration of Sankey diagrams for all four buildings.

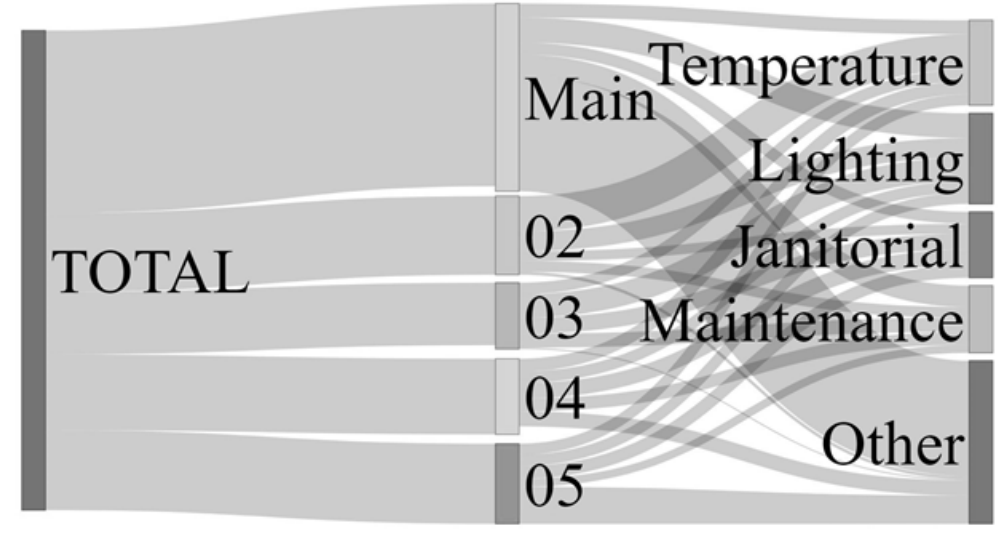

Figure 2.8: Example of a categorical and spatial breakdown of complaints in building two. 
Table 2.3: A Summary of floors with noted irregularities in the dataset.

\begin{tabular}{|c|c|c|}
\hline Building & $\begin{array}{l}\text { Floors } \\
\text { with irregular numbers of complaints }\end{array}$ & $\begin{array}{l}\text { Irregularity } \\
\text { description }\end{array}$ \\
\hline \multirow{5}{*}{ One } & 3 & $89 \%$ more complaints than floor average \\
\hline & 12 & $\begin{array}{l}79 \% \text { more complaints than } \\
\text { floor average }\end{array}$ \\
\hline & 6 & $\begin{array}{l}40 \% \text { more complaints than } \\
\text { floor average }\end{array}$ \\
\hline & 5 & $\begin{array}{l}68 \% \text { lighting } \\
\text { complaints }\end{array}$ \\
\hline & 13 & $\begin{array}{l}54 \% \text { lighting } \\
\text { complaints }\end{array}$ \\
\hline \multirow[t]{2}{*}{ Two } & 2 & $\begin{array}{l}38 \% \text { thermal and air } \\
\text { quality complaints }\end{array}$ \\
\hline & 3 & $\begin{array}{l}26 \% \text { lighting } \\
\text { complaints }\end{array}$ \\
\hline \multirow[t]{2}{*}{ Three } & Main & $\begin{array}{l}32 \% \text { maintenance } \\
\text { complaints }\end{array}$ \\
\hline & 12 & $\begin{array}{l}23 \% \text { janitorial } \\
\text { complaints }\end{array}$ \\
\hline \multirow[t]{2}{*}{ Four } & 4 & $\begin{array}{l}35 \% \text { janitorial } \\
\text { complaints }\end{array}$ \\
\hline & 8 & $\begin{array}{l}21 \% \text { thermal and air } \\
\text { quality complaints }\end{array}$ \\
\hline
\end{tabular}




\subsubsection{Analysis of the work-order logs}

\section{Frequency of key terms}

Terms in the dataset appearing with the greatest frequency can help pinpoint key trends/problems that operators face when they resolve complaints. Bar graphs were used to present the top 15 words used in the order of frequency in the dataset. Notably, the top issues the building operators encounter in each of the four complaint categories are replacing ballasts, adjusting temperature setpoints, assigning technicians, and unplugging toilets. Figure 2.8 represents the top 15 terms used by building operators when responding to complaints of the four studied categories for building one. The results from the frequency graphs display terms which are expected to be used for the corresponding complaint categories. For example, the most repeated words in the context of thermal and air quality complaints were "adjust", "thermostat", "air" and "vav". There does exist some overlap in terminology between complaint categories such as the term "toilet" being used commonly for both maintenance and janitorial complaints. However, it is important to look into the context of these words - maintenance com-

plaints regarding toilets also have the terms change, replace, leak and repair as the top terms while janitorial complaints have the terms unplug, replace, clean and paper. 

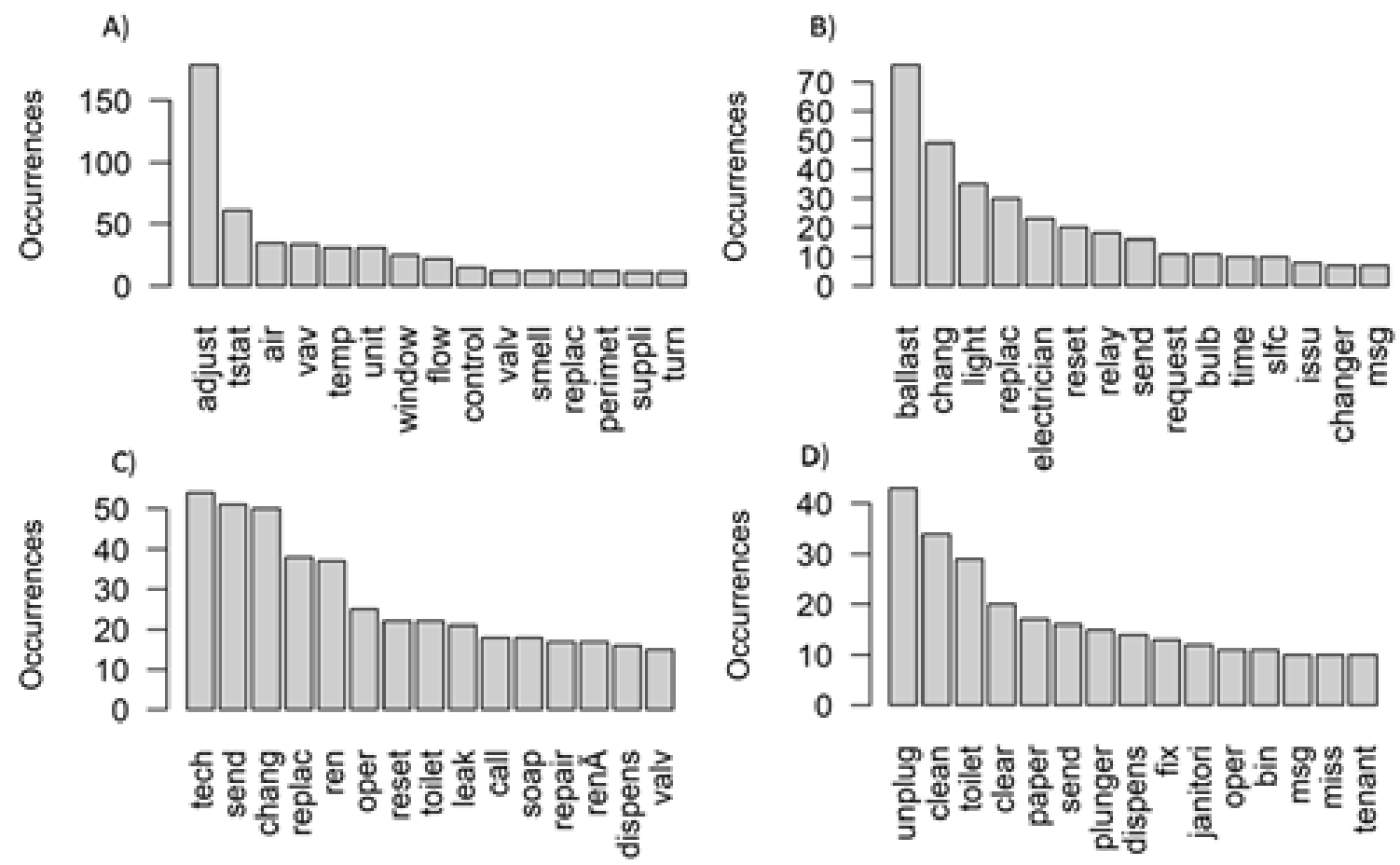

Figure 2.9: The top 15 terms used for a) thermal and air quality b) lighting c) maintenance and d) janitorial complaints.

\section{Association node networks}

Association node networks allow for the easy interpretation of the top rules identified by the ARM technique using the support/lift/confidence parameters. Larger nodes indicate a greater term frequency within the analyzed dataset. Darker nodes signify rules with a greater lift. Table 2.3 contains a list of the most prominent rules discovered when exploring the data sorted by complaint category for the entire dataset. Interesting rules which were observed included changing the toilet vacuum breaker mechanism and repairing the valve for perimeter heating units (window units) for maintenance-related complaints. Figure 2.9 shows a graphical representation of the rules that were discovered when exploring the irregularities found in maintenance related complaints on floor 12 of building one. This is a subset of the maintenance-related rules found in the maintenance category of Table 
2.3. It is evident that looking at the maintenance-related complaints in building one that operators are frequently changing the batteries on vacuum breakers (inside toilets), replacing the valves on the window unit heaters and tightening toilet seats. Repetitive use of these algorithms is required on real world datasets in combination with a theoretical understanding of their back end in order to generate meaningful insights. Often colloquialisms are not included within the pre filtering process which may make the first several runs of this algorithm generate rules which are not meaningful. Additionally, there is a trial and error component to optimizing the algorithm parameters such that insightful results are obtained.

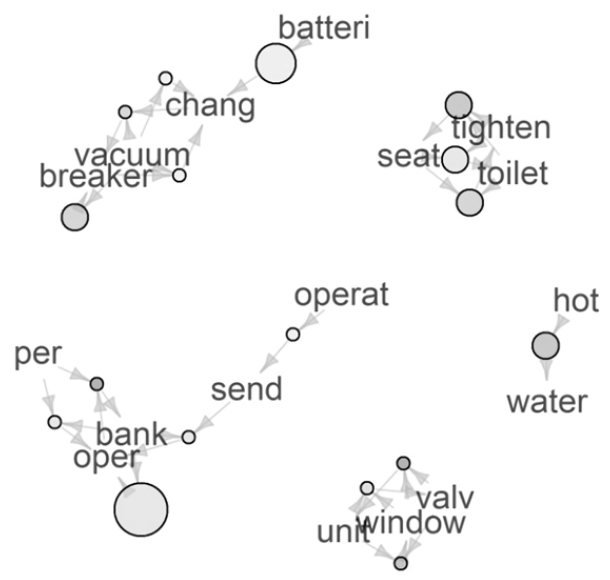

Figure 2.10: Association rules for maintenance related complaints in floor 12 of building one. 
Table 2.4: A Summary of floors with noted irregularities in the dataset.

\begin{tabular}{|c|c|}
\hline Complaint Category & $\begin{array}{c}\text { Top Rules Based on Operator } \\
\text { Comments }\end{array}$ \\
\hline lighting & $\begin{array}{l}\text { Replace } \rightarrow \text { Ballast } \\
\text { Reset } \rightarrow \text { Relay } \\
\text { Change } \rightarrow \text { Light } \\
\text { Send } \rightarrow \text { Electrician }\end{array}$ \\
\hline Thermal and air quality & $\begin{array}{c}\text { Change } \rightarrow \text { Thermostat } \\
\text { Set } \rightarrow \text { Temperature } \\
\text { Check } \rightarrow \text { Smells }\end{array}$ \\
\hline Janitorial & $\begin{array}{c}\text { Unblock } \rightarrow \text { Toilets } \\
\text { Clean } \rightarrow \text { Carpet } \\
\text { Call } \rightarrow \text { Plumber } \\
\text { Provide } \rightarrow \text { Paper Towel } \\
\text { Mop } \rightarrow \text { Floor } \\
\text { Remove } \rightarrow \text { Garbage }\end{array}$ \\
\hline Maintenance & $\begin{array}{c}\text { Change } \rightarrow \text { Vacuum Breaker } \\
\text { Change } \rightarrow \text { Battery } \\
\text { Valve } \rightarrow \text { Window Unit } \\
\text { Tighten } \rightarrow \text { Toilet Seat } \\
\text { Test } \rightarrow \text { Elevator } \\
\text { Call } \rightarrow \text { Elevator Company }\end{array}$ \\
\hline
\end{tabular}




\subsubsection{Unresolved issues and future work}

The CMMS analytics method presented in this study has several limitations that need to be addressed as future work:

- The method consolidates a large underutilized data source (i.e., complaint and work-order logs) to a form that is suitable for human interpretation. We envision that metrics derived by this method can be used to understand how operational resources are used, to identify abnormal floors, equipment, and work-order categories, and to improve operational performance. However, we did not investigate how facility managers use these metrics in practice. As future work, we will design a survey and conduct interviews with operators and facility managers to better understand how they use operational performance metrics.

- The availability of CMMS data in existing buildings differ substantially. For example, severity of the work-orders was not a routinely maintained field in the buildings of this study. In other facilities, operator work-order descriptions can be more or less detailed which may hinder the transferability of the method to other buildings. Future research is needed to standardize the CMMS fields and operational data collection practices in large commercial and institutional buildings.

- An issue that is yet to be resolved is the definition of the ARM parameters when the Apriori algorithm is applied to datasets of different characteristics. Currently, the support, confidence and lift parameters for each building and floor need to be tuned manually to generate meaningful rules and association node networks. A future study is needed to assess the viability of automating this process such that the users do not need to tune the hyperparameters of 
the algorithm to every new dataset it is applied to. A future implementation of these metrics in a commercial environment would have preset parameters which could be used by facilities management teams without the need for expertise in text mining.

- The use of multiple terms by different operators in explaining the same issue introduces inaccuracies to text mining algorithms. In the future, the appropriateness of natural language processing algorithms should be examined so that synonymous terminology can be detected.

- Text mining using ARM is only one way to gather insights from the dataset of work-orders. Methods such as topic modelling (using Latent Dirichlet Allocation and Non-negative Matrix Factorization) can be used to find naturally occurring subtopics within the work-orders and natural language processing (using lexicon and classifier based sentiment analysis) can potentially allow for complaints to be categorized into a hierarchy of severity using pretrained complaint data. Future work will build on the methods used in this study and integrate new techniques to extract further information from CMMS data.

- This study presents a methodology and a workflow by which CMMS data can be extracted and analyzed in order to draw conclusions about tenant satisfaction and building performance. The metrics that are developed require significant knowledge of programming in order to reproduce. Future work will include the development of a software toolkit for text mining and visualization of CMMS data using the R package Shiny. The prototype software tool will be used to present these metrics to an operator or building manager with a simplistic user interface. The software tool will be deployed over a local server so that any employee with access to the building network will be able to view these metrics 
through an online browser-based system. A limitation of this study is the lack of feedback from building operators. Building operators were not interviewed prior to the release of this study in order to discuss their feedback of the metrics. Interviews with operators will be carried out after the software tool development phase and iterative improvements will be made to both the software tool and the metrics.

- Another potential limitation for the transferability of the findings of this study is the absence of a CMMS in other buildings. The study was feasible on the four selected buildings due to the availability of a CMMS with four years of complaint and work-order logs. Even though such systems are common in large commercial and institutional buildings, some commercial buildings may not have them. In the future, the adoption of CMMS will allow metrics like the ones developed in this study to be applied to a broader range of buildings.

\subsection{Conclusions}

This study demonstrates how complaint logs and operator comments stored within computerized maintenance management systems can be used to extract operational performance metrics. A categorical and spatial breakdown of the complaints shows that complaints are concentrated around only a few floors of a building. Furthermore, a detailed analysis of the floor-level complaints shows that only certain complaint categories contribute to a majority of the recorded complaints. Temporal breakdowns demonstrated that all four buildings experienced a peak complaint frequency for both hot and cold complaints around $12 \mathrm{pm}$. A detailed analysis of the thermal and air quality complaints for the buildings varying with seasonal changes demonstrated that 
the hot and cold complaints had distinctive frequency distributions, with hot complaints featuring a prominent secondary peak in complaint intensity. A decision tree trained using temporal and thermal variations found that the highest probability of thermal and air quality complaints occurred on only a very small number of days during the shoulder season. Following these findings, further insights were developed by systematically extracting information through a text mining algorithm to explain the root-causes of the floor-level anomalies. Text mining building-level operator work-orders with a per category breakdown also reveals several key areas of repeated complaints that are common to all the floors of a studied building.

The use of ARM technique provides facility managers a more detailed insight into the work that operators undertake by creating dependencies among terms likely to be related to each other in the dataset. The Apriori algorithm was used to create the association rules needed three tuning parameters: support, confidence and lift. These parameters had to be individually tuned for each building in order to create meaningful rules. The most frequent issues that operators faced were replacing ballasts, changing zone temperature setpoints, unblocking toilets, and changing the vacuum breakers in washrooms, respectively. The rules indicating the co-dependencies were graphically represented by using association node networks. This resulted in an intuitive way for non-technical personnel to interpret the insights revealed by the ARM algorithm.

This study contributes to the literature by developing a method for extracting operational performance metrics from CMMS data using established text mining and visualization techniques such as the ARM, survival curves, stacked line curves, Sankey diagrams and decision trees. Previously, only a few studies have explored the viability of using CMMS to benchmark operational performance of buildings. However, this 
study is unique in applying innovative techniques to develop a suite of operational performance metrics from common CMMS data categories. 


\section{Chapter 3}

\section{Insight extraction using tenant surveys}

\subsection{Introduction}

Occupant satisfaction is a critical parameter for facility managers and surveys are powerful tools which allow managers to obtain valuable data and insights from their tenants [25]. Energy managers often seek to implement energy efficiency measures, but the impacts of those measures on tenant comfort and satisfaction must be quantifiable. This is because while the priority for an energy manager may be energy savings; tenants are usually more concerned for their comfort. For example, occupant surveys for Green Star rated buildings in Australia were used to demonstrate that there were differences in the perceived benefits of green workspaces between management and employees [26]. It was found that certain energy saving measures implemented by the management led to tenant dissatisfaction and could be counterintuitive to management's perception of the implementation of green building programs. Recognizing such areas of dissatisfaction from tenants should be an important goal for energy managers as well, since occupant behavior has been shown to have significant impacts on the energy consumption of a facility [27].

The traditional mechanism to assess occupant satisfaction with the indoor environment is to conduct tenant surveys [9]. Tenant survey datasets contain valuable 
information to benchmark building operational performance and identify anomalies but the traditional process of hiring employees to read through survey data and extract insightful information can be time consuming and labour-intensive - this is specifically applicable to survey data from large commercial institutions which may span over several years and may contain thousands of entries. Advances in text mining techniques such as sentiment analysis, topic modelling and association rule mining play an important part in the feasibility of incorporating text analytics as a factor in analyzing the effectiveness of building operations and maintenance. The importance of monitoring occupant satisfaction and workplace productivity is critical for facilities management teams, and it is recognized by today's building standards. For example, WELL standard recognizes the importance of monitoring occupant comfort and recommends occupant comfort surveys in buildings with 10 or more occupants with a participation rate of at least $30 \%$ once per year [28]. If survey results are to play a key role in the way operators and facilities managers use tenant feedback to modify their workflows, new analytical workflows must be developed from existing techniques that allow them to extract insights from free text in an efficient and intuitive manner.

\subsection{Literature review}

The potential of using occupant-centric benchmarks at a high resolution has been a popular topic of research. Free-text occupant survey responses are a source of occupant centric data; however, they can be too large and unstructured for manual interpretation. Alternative data sources have been utilized by researchers in order to gain insights into occupant satisfaction. One of these alternative data sources 
is human resources databases. For example, Newsham et al. carried out data analytics on a large corporate human resources database to extract metrics such as absenteeism, sick days and employee job satisfaction and performance [13, 29]. They compared these occupant satisfaction metrics in 46 buildings - 13 of which were LEED certified; and demonstrated that manager assessed job performance was slightly greater in the LEED certified buildings than the rest. Despite these case studies demonstrating the use of human resources data to extract metrics for occupant satisfaction, due to privacy and security concerns it may be difficult for facility managers to gain access to these datasets.

Computerized maintenance management system data may also be used as a proxy to monitor occupant comfort levels as well as operator workflows and schedules. Advances in the CMMS technology have led to it being commonly used in most large commercial buildings. The rise in popularity of scripting languages such as $\mathrm{R}$ and Python have also made these large databases simpler to analyze [30]. A few studies have made use of CMMS data in building performance analysis in order to classify building-level work orders [21], analyze and model thermal complaint frequencies $[23,31]$, and calculate component-level fault-frequency rates [33]. CMMS data have also been utilized in order to create high resolution temporal and spatial performance metrics for large commercial buildings as well as to predict thermal complaint rates given temporal categorical variables (e.g., time of day, day of week) and outdoor temperature-based parameters [34]. In a recent study, two years of CMMS data from a variety of buildings (offices, schools, laboratories, etc.) have been text mined in order to extract common maintenance requests and the most common work order types and user complaint categories were documented [24]. In another study, historical work order data have been analyzed in order to explore the trends in frequent maintenance activities in educational institutions showing 
that the majority of work orders are related to HVAC, electrical, and maintenance activities [35]. Interestingly, the analysis does not indicate that older buildings have a higher number of work orders and concludes that there needs to be more research to better understand the work order frequencies in different building types. They also recommended better subcategorization of data stored within the CMMSs - e.g., electrical work orders can be categorized as lighting and switch issues.

The ability to control indoor temperature is known to improve perceived comfort $[25,36]$; and, occupants in many modern office buildings are offered a limited thermostat override capability (e.g., $\pm 2^{\circ} \mathrm{C}$ from a default setpoint). Thermostat overrides provide insights into the frequency of thermal discomfort instances (discomfort enough to trigger a setpoint change) and their zone-level breakdown as well as indoor conditions that trigger these events [37]. For example, Gunay et al. developed models to predict the frequency of setpoint change requests at different indoor and outdoor temperatures and explored the potential of applying setpoints which minimize the thermostat interactions [38]. However, the hypothesis that indoor conditions that minimize the frequency of adaptive actions are the personalized comfort conditions has yet to be proven [39]. Researchers need to demonstrate the viability of treating adaptive behaviours as a proxy to comfort.

Mobile applications (e.g., cozie) for smartwatches and phones can be used to facilitate the collection of comfort data. For example, in a case study, Jayathissa et al. collected 1460 responses from 15 occupants over a month by using a smartwatch application [40]. Tenants were prompted to provide thermal comfort feedback at five intervals during a workday. The insight from the resulting comfort feedback data was used in conjunction with the temperature data to separate tenants into four groups based on their likelihood of being satisfied based on a cooler or warmer 
setpoint. The results indicated four separate groups of users: those which are satisfied in cooler environments, those that are sometimes satisfied regardless of the temperature, those that are always satisfied, and those that are comfortable most of the time. The results of this analysis can be used in making operational decisions such as recommending different zones for occupants who belong to different comfort profiles and developing energy saving opportunities through personalized controls.

Because it is often more difficult to apply data analytics and machine learning techniques on text-based data compared to traditional numerical data sources, surveys commonly ask occupants to rate their comfort or satisfaction levels regarding a certain issue on a numerical scale. However, the downside of this method compared to free text surveys is the gap that exists between the survey creator and the survey respondent. Respondents may not get to fully express the underlying issues in a survey where they have no control over the fields of input. Free text surveys, however, are difficult to manually analyze (especially in large commercial and institutional buildings with many tenants / occupants) and require time and effort to interpret. Manual interpretation of text-based data to extract operational performance metrics can be impractical. This disparity grows larger with datasets of larger sizes. If we aim to incorporate text-based tenant surveys as performance indicators for tenant satisfaction and workplace productivity, more robust tools must be developed to either automate or significantly reduce the time and effort required to draw quantitative observations from these highly unstructured data sources. In this study, we are developing a text mining method to analyze the information contained within free text surveys and demonstrating the functionality of sentiment analysis and topic modelling algorithms on survey data from a commercial building in Ottawa, Canada. Text analytics is a process by which large bodies of textual data are analyzed 
to derive insights and identify patterns. It is generally comprised of three steps: preprocessing, text mining, and postprocessing. Text mining operations are the most significant aspect of text analysis and include techniques such as association rule mining (ARM), clustering, and pattern discovery. Text mining represents an untapped opportunity to extract insights from a survey dataset. Several studies have shown the value of having free text comments integrated within non-linear survey questions regarding comfort distributed among tenants, as these provide venues for tenants to provide their own perspective to the facilities management team [41]. However, textual data tend to be overlooked in the field of building performance as their analysis requires tools which till recently were not widely available [42]. To this end, this study presents a natural language processing-based text mining method to extract operational insights from tenant surveys. The method is demonstrated in a case study building. The effectiveness of several text mining techniques is examined, their shortcomings are identified, and future work recommendations are developed.

\subsection{Methodology}

As illustrated in Figure 3.1, The methodology involves three separate approaches to analyze survey responses. First, a visual approach is employed to compare the surveybased floor-level complaint distributions to the service requests recorded in a CMMS. This is intended to ascertain if the complaint patterns within a solicited source of feedback (surveys) would be representative of the complaint patterns. Secondly, a text mining approach is employed using ARM and two different topic modelling algorithms: Latent Dirichlet Allocation (LDA) and Non-Negative Matrix Factorization $(\mathrm{NMF})$. This is intended to extract key terms and topics found throughout the 
survey responses. Thirdly, a natural language processing approach is employed with seven different lexicon and classifier-based sentiment analysis algorithms to find the overall satisfaction of the tenants. The accuracy of these algorithms is calculated by comparing them to manually classified survey data. A step by step approach to this analysis can be found as open source code within the provided GitHub repository [43].

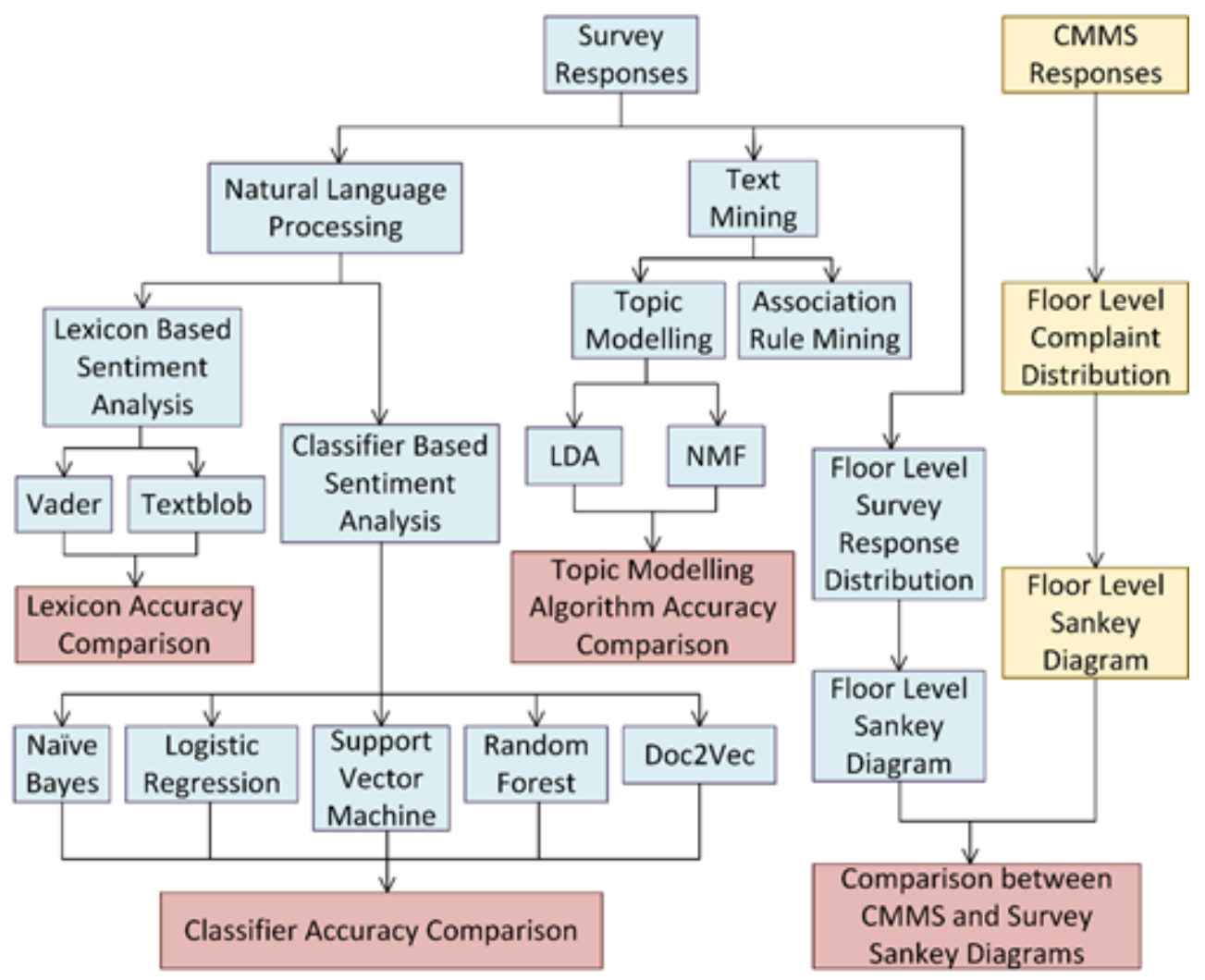

Figure 3.1: An overview of the workflow employed in this study.

\subsubsection{Characteristics of the dataset}

In this study, tenant survey data and CMMS data were extracted from a large commercial building (111,738 $\mathrm{m}^{2}, 19$ floors and 138 tenants) located in Ottawa, 
Canada. Survey response data is a form of solicited feedback (i.e. the tenants are prompted for feedback on their comfort) whereas CMMS data is a source of unsolicited feedback (i.e. the tenants log complaints themselves without any request from the facility managers). CMMS data was obtained from servers hosted by a third-party provider hired by the facility managers to catalogue tenant complaints, whereas surveys were completed by individual tenants at the end of a year to give them an opportunity to share their opinions with the facility. Tenants have the option to contact the third-party CMMS service through a phone call or online. Once a tenant logs a complaint, the CMMS operators generate a work order to address the issue. Building technicians are then asked to address the complaint and enter the description of the methodology they undertook to resolve the tenant's complaint.

There were 215 survey entries, with each survey entry prompting the tenant for feedback on thermal and maintenance related concerns. Hence, the total dataset had 430 surveys responses for analysis. The floor number corresponding to the rented space for each respondent in the survey was present alongside their survey response entry. These responses are samples of solicited feedback whereby the facility managers received suggestions. The two data fields used to compare similarities between the CMMS and the survey data are the complaint type (e.g. thermal complaints, maintenance complaints) and the floor number. The survey responses were typed out in the appropriate fields by individual tenants in the building through an online form. The results from that form were made available to the authors for research purposes by the facility managers. It is important to note the difference between the survey and CMMS datasets. While the latter has highly structured data entries allowing for easy data extraction and analysis, the survey responses provide only a high-level complaint category (thermal / maintenance), floor number, and the free form text which is more challenging to analyze than simple numerical entries. 
The ideal free text dataset would contain text based data relevant to the survey categories, with the text being non repetitive, brief and descriptive with a high number of key words and a lack of colloquialisms. Unfortunately such well structured text based data is rarely found in real world datasets and processing of the data is required before insights can be extracted from it. The following section contains a detailed description of the pre processing steps which were utilized in order to make the data suitable for analysis.

\subsubsection{Data preprocessing}

Data analytics tools in Python were used to extract the relevant fields from the survey response data and store the text fields into two separate categories; "thermal" and "maintenance" using dataframe manipulation libraries to split the two survey entry types into their own separate entries. All letters were converted to lowercase in order to prevent the text mining algorithms from mischaracterizing words spelt with a different case, and stop words were removed from the survey entries. Stop words are commonly used terms within a body of text that are usually removed prior to text analytics as they contain little to no useful information, e.g., "a", "the", "is". Irregular characters such as letters with non-English accents ("é", "ê", etc.,) were filtered out and html entries were decoded and reverted into plain text. Stemming was employed in order to reduce words to their root forms; for example, words such are "temperature" and "temperatures" revert back to the same root meaning but unless stemmed will be registered as two separate words by the analytical algorithm and may skew the results of the analysis. In this case the porter stemming algorithm would reduce both terms to the root term: "temp". Stemming allows for the text analytics algorithms to recognize and group together words with the same roots but 
different suffixes.

The text preprocessing stage is critical to an accurate analysis as the presence of stop words and similar words with mismatching cases can detrimentally affect the performance of ARM and topic modelling algorithms. CMMS data were analyzed in order to group the thermal and maintenance complaints on a per floor basis. The data were read and incomplete entries in the database (i.e., mislabelled categories, floors etc.) were removed from the dataset. The complaints belonging to the categories "Thermal" and "Maintenance" were filtered out into a new data frame and a matrix of the per floor complaint distributions for the complaint categories "Thermal" and "Maintenance" were created. The matrix was used to build a Sankey diagram for the unsolicited complaint distribution demonstrating the spatial and categorical patterns in the distributions of the complaints. An identical methodology was followed with the tenant survey dataset and a similar spatial and categorical Sankey diagram was generated using it. The top five floors where the highest numbers of complaints were recorded and compared between both datasets.

\subsubsection{Text mining algorithms}

Text mining algorithms were employed on the tenant surveys in order to discover hidden semantics using unsupervised learning techniques. ARM is a text mining technique to identify frequent item sets within a dataset by using the Apriori algorithm. In this study, we employed the ARM technique to identify word combination patterns that were repeatedly used in describing work orders. The most important

parameters while dealing with ARM are the support, confidence and lift of the association rules. Support is defined as a measure of the proportion of instances 
in a dataset that an itemset appears (e.g. fraction of work orders in which several terms co-exist). A high support value for a rule is an indication that the rule exists among multiple entries in the dataset. The second metric confidence represents the proportion of work orders with the word X where the word $\mathrm{Y}$ also exists. A high confidence value indicates the level of confidence that an itemset does not exist based on pure coincidence. Hence, it is favourable to find itemsets with high support and confidence levels. The third metric lift is a measure of the probability of a rule appearing together in a dataset divided by the product of the probability that the terms making the rule appear separately. A lift value greater than one suggests that occurrences of words X and Y are dependent on each other. The Apriori algorithm was used to generate a list of word coexistences with pre-defined support and confidence thresholds. The ARM method is applied on the tenant survey responses, and the frequently co-occurring terms for the thermal and maintenance-related comments are graphed and highlighted.

Topic modelling is an unsupervised approach to discovering repetitive "topics" or "themes" which occur frequently in bodies of text. LDA is used to model topics in a document. Documents with similar topics use similar groups of words. Latent topics are discovered by groups of words in a text corpus that occur together within documents. While a simple bag of words model focuses on word frequencies, LDA uses probability distributions. LDA creates a statistical model for discovering abstract topics occurring in a collection of documents [44] allowing for the extraction of key features and information from an otherwise difficult to analyze data medium. NMF is a feature extraction algorithm which separates out contextual patterns within a body of text. NMF takes in a document term matrix (DTM) as input where each column represents a document, each row of the column indicates the weight of a word contained in the document. It creates a set of topics based on weighted 
sets of co-occurring terms by breaking down the original DTM and contributing the attributes until contextual features are found. For example, the terms hot and cold may appear in a document related to complaints about washrooms in relation to complaints regarding the temperature of the tap water as well as in complaints about heating and cooling setpoints causing thermal discomfort. Each of these would be recognized by NMF as a separate context and the separate topics would be created. Both the NMF and LDA algorithms are applied to each set of tenant complaints. After a sensitivity analysis process assessing the interpretability of the topic outputs as a greater number of topics were added to the algorithm, it was determined that six topics were optimal for the analysis.

A lexicon-based sentiment analysis algorithm consults a standard database of commonly used words. Each word carries a positive or negative connotation and contributed to the overall polarity (positivity / negativity) of a body of text. Searches for negations are added to lexicon-based sentiment analysis algorithms which allow for a sentiment to be reversed if a negation such as "not" is placed before an adjective contributing to overall polarity [45]. Lexicon based sentiment analysis methods are computationally less intensive and can be useful in deciphering polarity for shorter bodies of text. They also do not require manual training which decreases the amount of effort put into the analysis of a textual dataset. The disadvantage of this method is the dependence on standard dictionaries of words to carry out sentiment analysis. While this method may be applicable for analysis on generic comments, terminology specific to HVAC systems in buildings may not be recognized and their contributions to the overall polarity of a sentence can be lost. It is possible in most algorithm libraries to manually add specific terminologies in order to obtain a more accurate sentiment, however, doing to adds time to the analytical workflow. For the purposes of this study, we employed two lexicon-based sentiment 
analysis packages (Vader and TextBlob) available in Python. Textblob and Vader were chosen for the purpose of this analysis since they are commonly used libraries and are two of the most widely established and used lexicon-based sentiment analysis approaches used in the field of text mining. The accuracy of both algorithms with respect to identifying negative and positive comments was compared to each other and to other sentiment analysis methods. In order to evaluate the accuracy of the lexicon-based sentiment analysis algorithms, the dataset was manually classified into a binary score (neutral / negative) and the polarity score output from the survey comments was converted to a similar binary score. The binary classification was defined from neutral to negative as only an insignificant fraction of the comments within the dataset (less than 1\%) were found to be positive. This was expected as tenants completing these surveys are usually making their concerns associated with the building heard, and not reporting their positive experiences. All polarity values zero or less were considered to be negative while the polarity values greater than zero were considered to be neutral. The output from the lexicon algorithms was then compared to the manual classification and a percent accuracy for the derived classes was calculated.

Classifier based sentiment analysis techniques make use of supervised machine learning. The dataset is divided into two predefined fractions: training and validation. A pipeline of operations is defined where each entry within the dataset (containing text-based survey responses) is first vectorized and then turned into a term frequency-inverse document frequency (TF-IDF) format. The training set is classified binomially (neutral / negative) and used to train the classifiers. The trained classifier is used on the validation set in order to obtain insights on the dataset. The advantage of a classification-based approach to sentiment analysis is that the classifier can be trained to identify specific terminology for specific datasets such as 
thermal and lighting complaints in a building. This lends to a specialized solution for sentiment analysis compared to a lexicon-based approach. The disadvantage of classifiers is that they take time and effort to train compared to lexicon-based approaches. Six different classifiers were trained and validated on the dataset: naïve Bayes, logistic regression, decision tree and random forest, support vector machines, and Doc2Vec classifiers.

The naïve Bayes classifiers (Python: sklearn, MultinomialNB) use the Bayes' theorem which defines the probability that an event will occur, given prior knowledge of the conditions that may be related to the occurrence of the event. A naïve Bayes classifier will split up all the words of a survey response in the training set and assign a probability for each word contributing to the outcome of the survey response being positive or not being positive. The per sentence probability for being either negative or neutral is then computed and the decision with the highest probability is selected as the class which the sentence belongs to. In this way, we assume that each term found within the sentence is a feature and the presence of these features is what dictates the outcome (classification) of being negative or neutral. The naïve Bayes algorithm, however, has the downside of assuming that the features being used to predict the class are unrelated to each other and so does not take into account the possibility that the features themselves may depend on the existence of others. Since it is a very well established algorithm, it is often used as a baseline for classifier accuracy when studies are performed with multiple classification techniques in order to study the validity of other classification algorithms [46].

Logistic regression (Python: sklearn, LogisticRegression) allows for a binary outcome prediction given multiple independent variables or features that determine the outcome. Logistic regression will determine the relationship between these independent 
features and the output variable through the use of a sigmoid function to estimate the probability of the outcome belonging to one of the two binary classes. Logistic regression also fits coefficients to each of the independent features which allow for the interpretation of relative importance that each input has for the model.

Support Vector Machines (SVM) are supervised machine learning algorithms which are often applied to classification and categorization problems [47]. A hyperplane is a divider between two sets of data points. A support vector machine (Python: sklearn, SGDClassifier) is an algorithm which segregates the two classes using hyperplanes. Support vector machine algorithms will try to maximize the margin between data points and the hyperplane to find the most suitable areas where these planes can be inserted. Hyperplanes can be linear, nonlinear or three dimensional. In order to use a support vector machine for classification, the documents must be turned into vectors. This is done on a per sentence basis where a TF-IDF is generated. The dataset is then mapped into a space where a hyperplane is drawn between the vectors separating them into two distinct classes.

Decision tree classifiers used in this study are classification trees as they predict the likelihood of a comment belonging to one of two binary classes. Decision trees split into nodes, each of which correspond to various factors influencing the outcome and leaves which are the outcomes themselves (binary neutral / negative in this case). The nodes keep creating splits until externally applied stopping criteria are reached. The downside of a decision tree is that it is easy to overfit the data and create non representative models. A random forest classifier works in a very similar fashion to a decision tree; however, it generates several trees from randomly selected subsets of data and averages the results in order to have a more representative fit of the entire dataset. This usually results in a much better model which does not as easily overfit. 
Doc2Vec (Python: Genism, Doc2Vec) is a supervised classification algorithm that builds on the Gensim Word2Vec package. Word2Vec is based on an algorithm called "skip gram" which represents words in training sentences as vectors and predicts the probability of surrounding words given a central term [48]. By expanding this concept out to have an additional vector involved in a per document basis called the document vector, sentences can be trained for semantic context (positivity / negativity) as well. It is a neural network trained to recognize the impact on document polarity caused by the presence of certain words.

\subsection{Results}

\subsubsection{Comparison of solicited and unsolicited complaint fre- quencies}

Both surveys and CMMS entries contain complaints. They are differentiated in their means of collection. Whereas CMMS complaint entries are collected continuously throughout the year, survey responses are collected at the end of the year. As shown in Figure 3.2, a visualization of the complaint and survey response distributions per floor demonstrates that three of the five floors with the highest rate of complaint rates from survey responses and CMMS were identical. This can be considered a preliminary evidence for the usability of unsolicited data (CMMS) in lieu of solicited data (surveys). Future research is needed to explore the use of unsolicited occupant feedback (thermal complaints logged in a CMMS or thermostat interactions logged in a building automation system) instead of surveys to infer occupant preferences and

satisfaction. An additional observation is that tenants that seem to be more willing 
to write about thermal issues in surveys but rarely complain about them in the CMMS dataset where only a very small fraction of the complaints are about thermal issues. The results may be an indicator that tenants have different thresholds to complain via CMMS and surveys. In surveys, tenants are more likely to provide a holistic view of their thermal comfort and maintenance related issues. This is in contrast to tenants issuing complaint calls at more severe discomfort levels which may be more frequent for maintenance related issues compared to thermal issues.
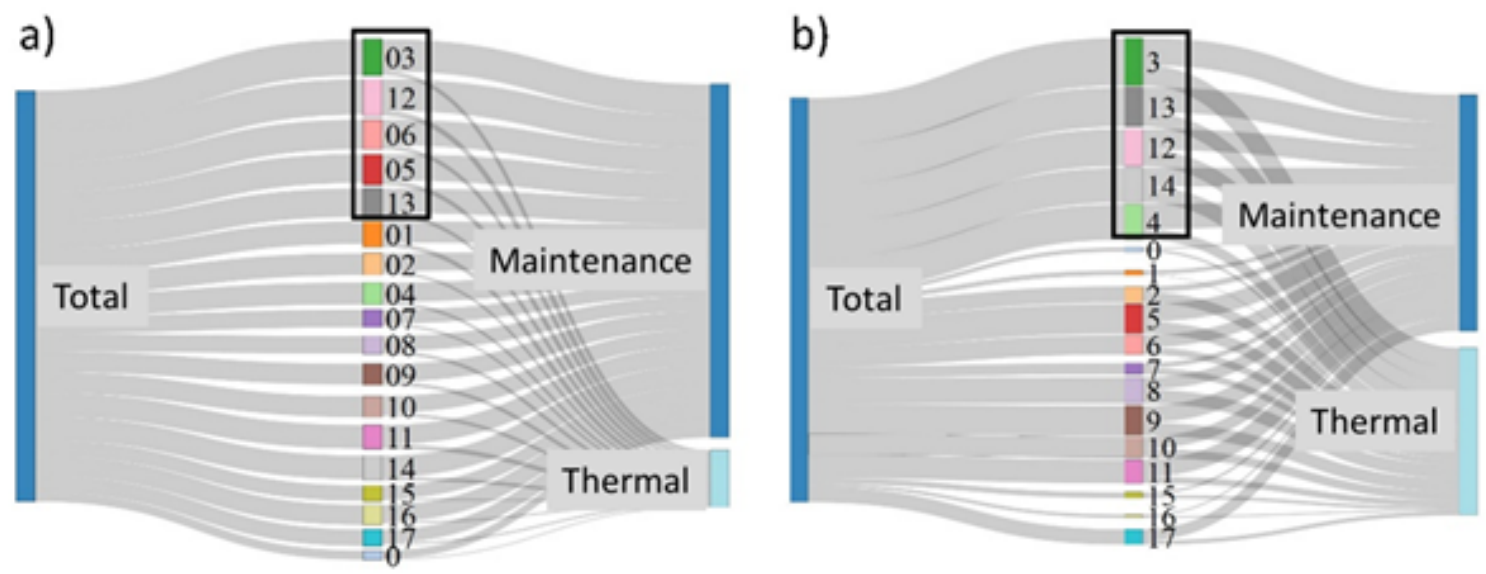

Figure 3.2: A comparison between the per floor breakdown of thermal and maintenance complaints from (a) CMMS and (b) survey responses.

\subsubsection{Text mining algorithms}

\section{Association rule mining}

Association node networks allow for the easy interpretation of the top rules identified by the ARM algorithm, see Figure 3.3. Larger nodes indicate a greater term frequency within the analyzed dataset. Darker nodes signify rules with a greater lift. The ARM algorithm was applied to the thermal and maintenance-related survey 
responses and the most frequent rules that were derived were displayed. Interesting observations include that tenants were concerned about air quality, heating on Mondays, thermostats, and having greater control over the thermostats in the winter in their thermal survey responses while they were concerned about a lack of water in the washroom taps, empty soap dispensers and a lack of car parking in the maintenance survey responses. A downside of free text surveys can be observed here, as tenants frequently reported being cold within the maintenance section of the survey; resulting in the ARM algorithm picking that group of complaints up in a section where it should be irrelevant. Inaccuracies in these algorithms usually stem from terms which are irrelevant to insight extraction such as names of staff being included within the dataset and not being part of the pre-processing methodology. Therefore, it is often required to run these algorithms multiple times and extract irrelevant terms which the pre-processing steps initially failed to remove manually. 

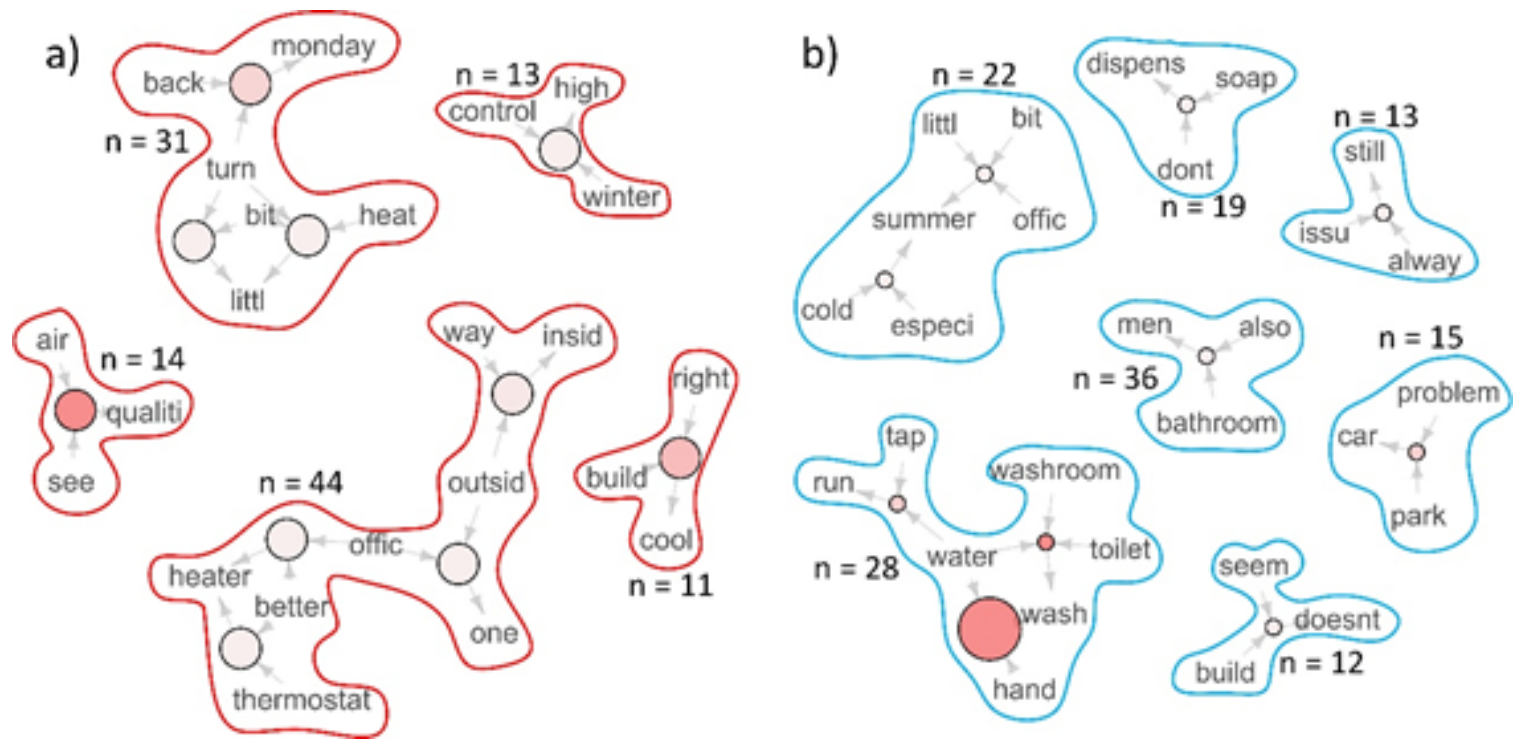

Figure 3.3: Association node graphs built using ARM. The figure contains rules derived for (a) thermal and (b) maintenance-related survey responses. Rules which co-occurred frequently are grouped together and the total number of occurrences for each rule group is denoted by $n$.

\section{Topic modelling}

Topic modelling algorithms LDA and NMF are employed in order to use an unsupervised learning approach towards finding groups of similar complaint topics. The top six topics are selected for each of the categories (thermal, maintenance) and results from both algorithms are presented in Table 3.1. There is a balance involved in the generation of topics through LDA and NMF. A high number of topics has the potential to cover a wider range of topics contained within the dataset, but distinctness between groups decreases as the topics are increased. In other words, if too many topics are created for the model to derive, there is an overlap between word groups of different topics making the interpretation of the results difficult. Iteratively generating topic models with three to ten topics demonstrated a lack of interpretability for more than six topics using the dataset. Examples of complaints represented by each topic for both survey categories and algorithms are represented 
in Tables 3.2 and 3.3. It was found that certain topics (heating on Mondays, air circulation, lack of washroom soap, parking issues) found from this analysis were in line with those identified by the ARM technique. Further interesting observations include feedback regarding tenants desiring access to stairs, better control of thermostats, a high frequency of elevator breakdowns, a desire for better food options, complaints due to a nearby subway construction and issues regarding revolving doors. 
Table 3.1: The six topics derived from the thermal and maintenance survey responses using the LDA and NMF algorithms.

\begin{tabular}{|c|c|c|c|c|c|}
\hline \multicolumn{6}{|c|}{ LDA Thermal Surveys } \\
\hline Topic 0 & Topic 1 & Topic 2 & Topic 3 & Topic 4 & Topic 5 \\
\hline think & know & sure & think & turn & control \\
\hline air & don't & temperature & office & heat & air \\
\hline probably & air & really & yet & cold & temperature \\
\hline circulation & heating & don't & window & idea & floor \\
\hline better & conditioning & know & sure & think & would \\
\hline \multicolumn{6}{|c|}{ LDA Maintenance Surveys } \\
\hline Topic 0 & Topic 1 & Topic 2 & Topic 3 & Topic 4 & Topic 5 \\
\hline elevators & people & building & paper & soap & often \\
\hline floor & stuck & could & can't & would & would \\
\hline slow & cleaners & cold & washrooms & like & floor \\
\hline need & clean & would & people & floor & work \\
\hline one & elevators & revolving & floors & elevators & time \\
\hline \multicolumn{6}{|c|}{ NMF Thermal Surveys } \\
\hline Topic 0 & Topic 1 & Topic 2 & Topic 3 & Topic 4 & Topic 5 \\
\hline know & turn & cold & think & air & sure \\
\hline don't & heat & $\mathrm{ac}$ & temperature & conditioning & operates \\
\hline really & ac & make & control & circulation & rectify \\
\hline reason & sooner & warm & building & needs & difficult \\
\hline idea & fall & really & would & summer & reasons \\
\hline \multicolumn{6}{|c|}{ NMF Maintenance Surveys } \\
\hline Topic 0 & Topic 1 & Topic 2 & Topic 3 & Topic 4 & Topic 5 \\
\hline floor & elevators & going & stairs & cold & would \\
\hline soap & slow & people & can't & water & nice \\
\hline bathrooms & stuck & building & use & sometimes & food \\
\hline often & get & construction & go & temperature & like \\
\hline washrooms & people & lrt & floors & always & building \\
\hline
\end{tabular}


Table 3.2: Examples of thermal-related tenant survey responses.

\begin{tabular}{|l|l|l|}
\hline Survey response & LDA Topic & NMF Topic \\
\hline $\begin{array}{l}\text { I'm guessing it's because of the thermostat, it's just on high } \\
\text { of a temperature or too much humidity. It's quite a bit of a difference } \\
\text { coming in from outside to inside. }\end{array}$ & 0 & 5 \\
\hline $\begin{array}{l}\text { I think there is poor circulation and it would be better if } \\
\text { there were more fans or air filtering. }\end{array}$ & 5 & 1 \\
\hline $\begin{array}{l}\text { Generally, it should be regulated more with what is going on } \\
\text { outside but when the temperature is cooler maybe it changes but we are } \\
\text { wrapped up in wraps and I just took my wool wrap off. }\end{array}$ & 4 & 0 \\
\hline $\begin{array}{l}\text { I think it's just finding a balance between the temperatures } \\
\text { especially in fall and in spring when it's hard to find that balance. I guess } \\
\text { just playing around with temperature controls. }\end{array}$ & 5 & 0 \\
\hline $\begin{array}{l}\text { It would be good to have a little bit more control over your } \\
\text { area. Where I am, I don't have any controls, I don't have a thermostat in my } \\
\text { area. }\end{array}$ & 2 & 4 \\
\hline
\end{tabular}


Table 3.3: Examples of maintenance-related tenant survey responses.

\begin{tabular}{|c|c|c|}
\hline Survey response & LDA Topic & NMF Topic \\
\hline $\begin{array}{l}\text { Just because this building is energy efficient doesn't mean that } \\
\text { it has any type of sustainability for the outside environment. There is no } \\
\text { consideration for the birds hitting the windows and there is no consideration } \\
\text { for the amount of traffic congestion and the amount of emissions that are } \\
\text { emitted from the cars going up and down Bank Street that people are exposed } \\
\text { to. So, this building while it may be green, or LEED certified it's really } \\
\text { not. }\end{array}$ & 4 & 5 \\
\hline $\begin{array}{l}\text { I think the biggest thing I notice is the lights and I can't } \\
\text { believe how much are on the weekends when I come in and all the lights are on } \\
\text { in the entire office when nobody is there. I'm just wondering how we can } \\
\text { possibly be a Green building when that's happening and there are many other } \\
\text { buildings that do have models that we might want to look into, because all } \\
\text { the lights on the weekends when nobody is around makes no sense. }\end{array}$ & 0 & 5 \\
\hline $\begin{array}{l}\text { The washrooms on the 2nd floor, the women's washroom } \\
\text { especially is used by a lot of the public not just the tenants so a lot of } \\
\text { the times the cleanliness of the washrooms is questionable. The replenishment } \\
\text { of toilet paper or paper towels sometimes is not as good as it should be. }\end{array}$ & 5 & 0 \\
\hline $\begin{array}{l}\text { The men's bathroom on the 13th floor that have } \\
\text { malfunctioning taps and soap dispensers. During the day the bathrooms are not } \\
\text { always very clean. I don't have anywhere to park, so I have to take the bus. }\end{array}$ & 0 & 0 \\
\hline $\begin{array}{l}\text { We can't go between floors using the stairs, we have to use the } \\
\text { elevator. I have to use the elevator and I'd rather walk. }\end{array}$ & 3 & 1 \\
\hline
\end{tabular}




\section{Lexicon based sentiment analysis}

Recall that lexicon-based sentiment analysis uses a standard dictionary of term with predefined polarity values. It then adds modifiers to the polarity values of individual terms by taking into consideration suffixes and prefixes, calculating an overall sentiment value per survey response. These sentiment polarity scores have been broken down on a per floor basis with the mean polarity, maximum polarity and minimum polarity being demonstrated in Figure 3.4. Only the results form the Textblob library are shown here as the Vader results are fairly similar and do not demonstrate further insight into the complaint patterns.
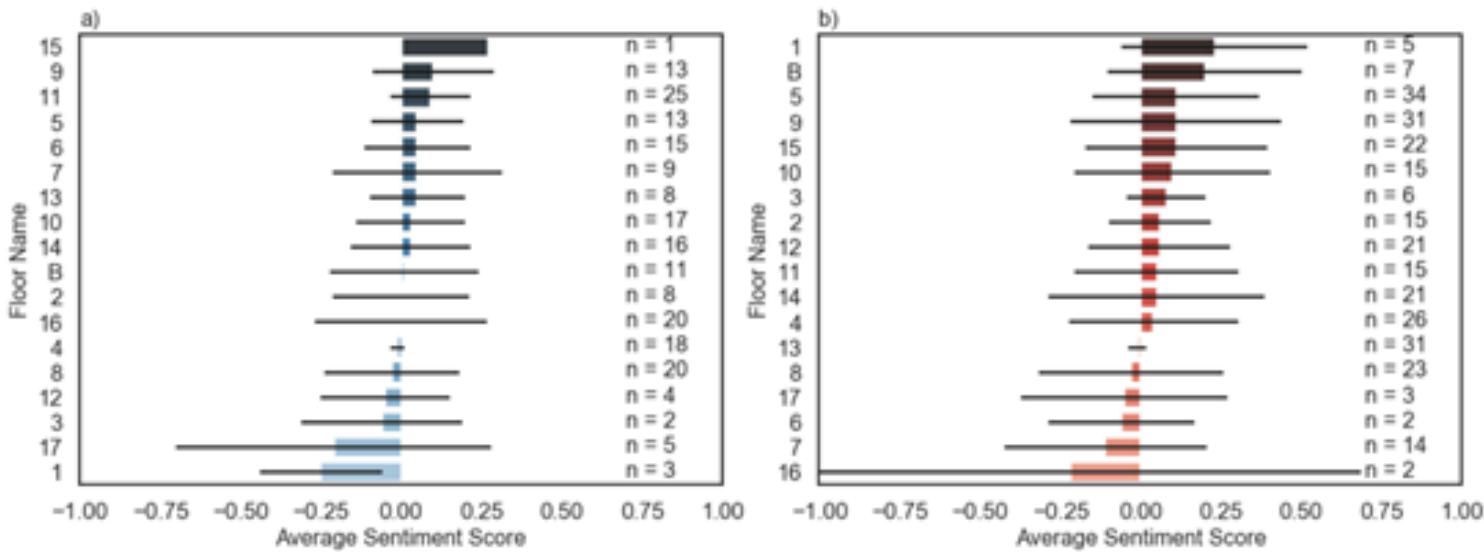

Figure 3.4: Average sentiment score and standard deviation of the survey responses for (a) thermal feedback and (b) maintenance feedback on a per floor basis using the TextBlob sentiment analysis library. The number of survey responses per floor is denoted by $n$

Both the TextBlob and Vader sentiment analysis algorithms were applied to the dataset in order to obtain a distribution of sentiment scores across the floors. Recall that the survey entries were manually classified as neutral and negative for the validation of the algorithm into two categories. In order to assess the validation 
of the lexicon-based approaches, the histogram of polarity for all complaints were plotted alongside those for only negative comments. As shown in Figure 3.5, both histograms showed a similar shape indicating that the lexicon-based approach had failed to accurately classify the complaints.
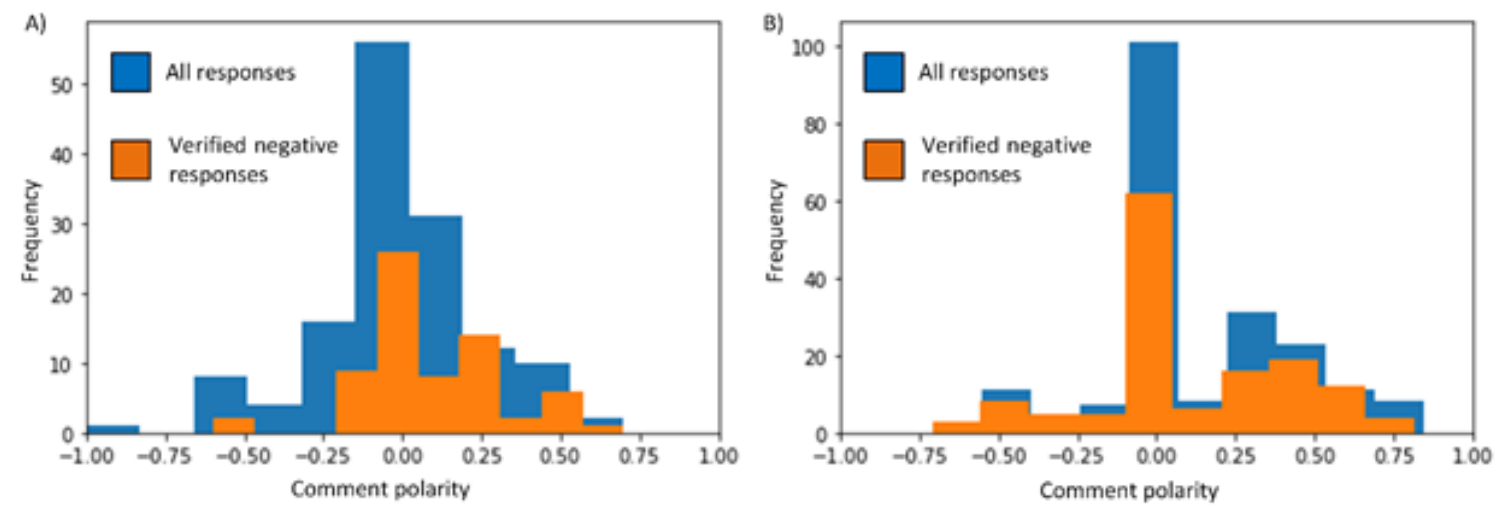

Figure 3.5: Histograms of the response polarity distribution for all survey responses compared with the polarity distribution for survey responses manually verified as negative by the (a) TextBlob and (b) Vader algorithms.

An example of the survey responses and their sentiment scores as obtained directly from the TextBlob algorithm are presented in Table 3.4 and Table 3.5 for thermal and maintenance-related issues, respectively. The validated scored have been determined by the researchers and are used to verify the validity of the polarity scores obtained by using the algorithms. Recall that the polarity output from the lexicon-based sentiment analysis algorithms varied between -1 and 1 . In order to evaluate the accuracy metric of these algorithms, the polarity output was converted to a binary scale (negative if polarity was less than or equal to zero and neutral if polarity was greater than zero). The accuracy for the TextBlob and Vader classifiers were found to be $33 \%$ and $45 \%$, respectively which were the lowest in the study. It is possible that lexicon-based algorithms are more suited to generalized datasets 
instead of domain specific ones regarding HVAC and maintenance-related complaints.

Table 3.4: Examples of survey responses to thermal issues and their polarity.

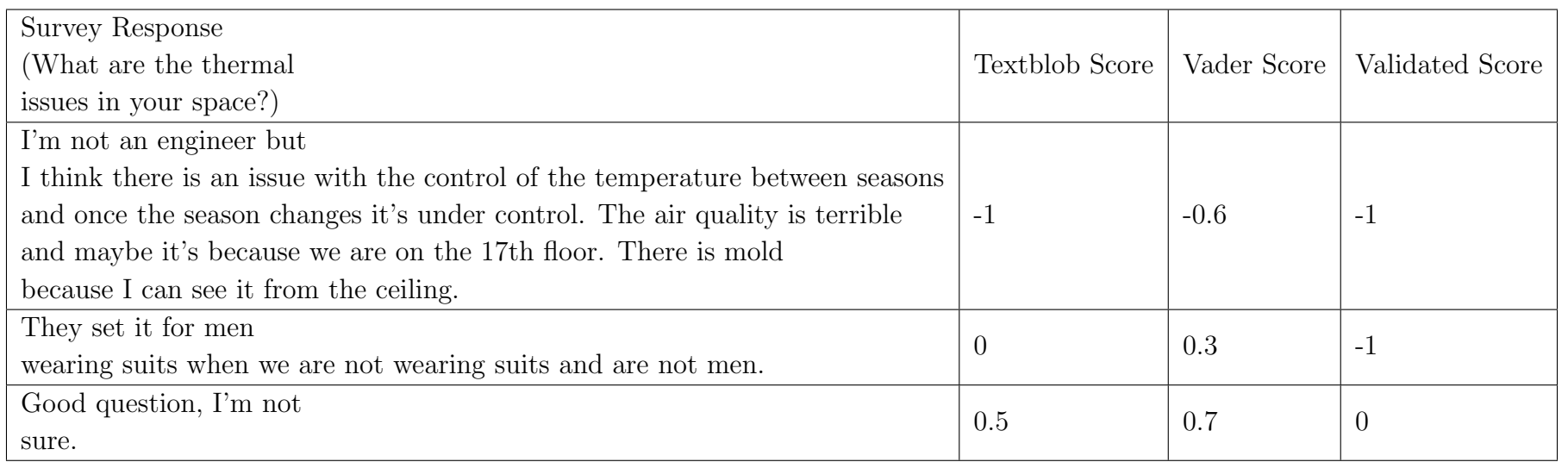

Table 3.5: Examples of survey responses to maintenance issues and their polarity.

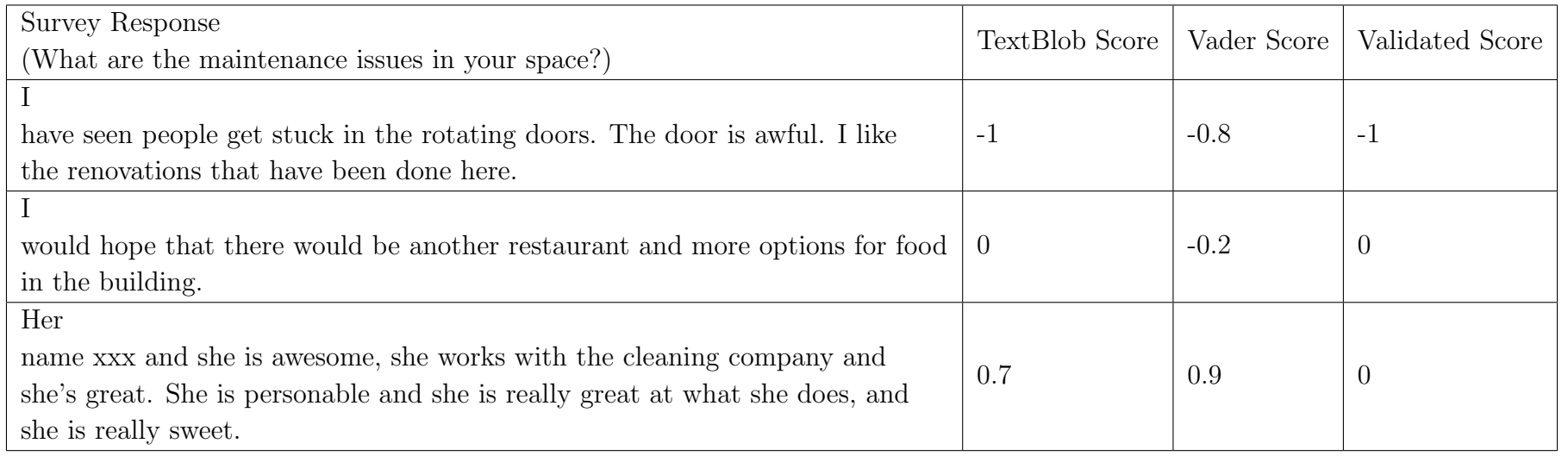

\section{Binomial classifiers}

Recall that binary classification uses a supervised approach to machine learning in order to make a binary prediction about the class a response belongs to (in this case the classes are negative and neutral). Here the approach is to have a training and validation set where $70 \%$ of all the data is used for training and $30 \%$ is used in the 
validation approach, see Figure 3.6. A higher fraction of the dataset was used for the training set (70\%) compared to the validation set (30\%) than the usual approach in training models. This approach was used as when experimental training was performed on $30 \%$ of the dataset and validation on $70 \%$ it was found that the models were highly prone to overfitting due to the limited dataset size and a large negative bias of the data. Free text data from the tenant surveys were extracted, preprocessed and the sentiments of the survey responses were classified manually into a binary (neutral / negative) group. $70 \%$ of the data was used to train a variety of classifiers (naïve Bayes, Logistic Regression, Support Vector Machine, Doc2Vec, RNN). Once trained, the validation survey comments were applied to the classifier and the classification results were compared to the manual classification. A percentage accuracy was calculated for each classifier in order to assess its performance. It should be noted that the methodology used in this study is not proposed as a guide to a workflow for using these methodologies. It is meant to be a quantitative assessment of a holistic range of methods that can be deployed in order to extract meaningful insights from unstructured text-based data. It is up to the reader to assess which techniques are suitable to their own analytical purposes. The objective of this study is to demonstrate the potential of a wide range of text mining methods on text-based survey data. 


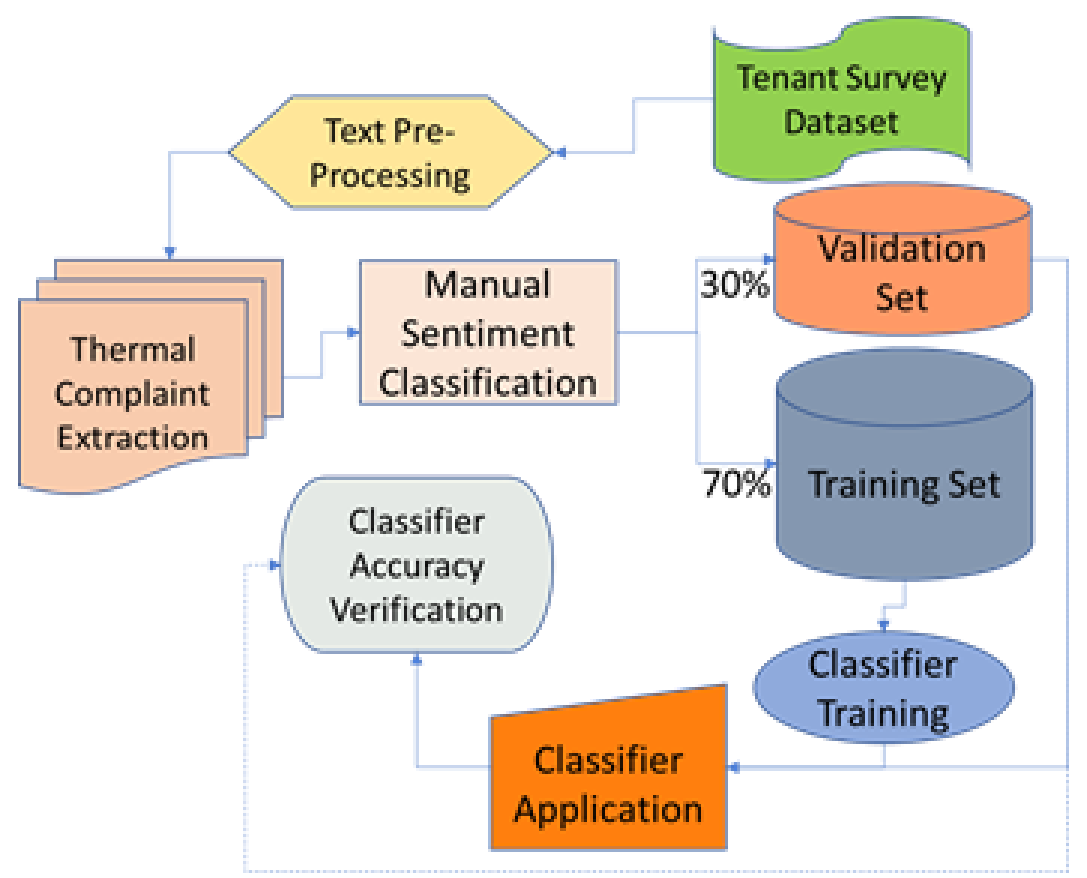

Figure 3.6: An overview of the operations pipeline during binomial classification. 

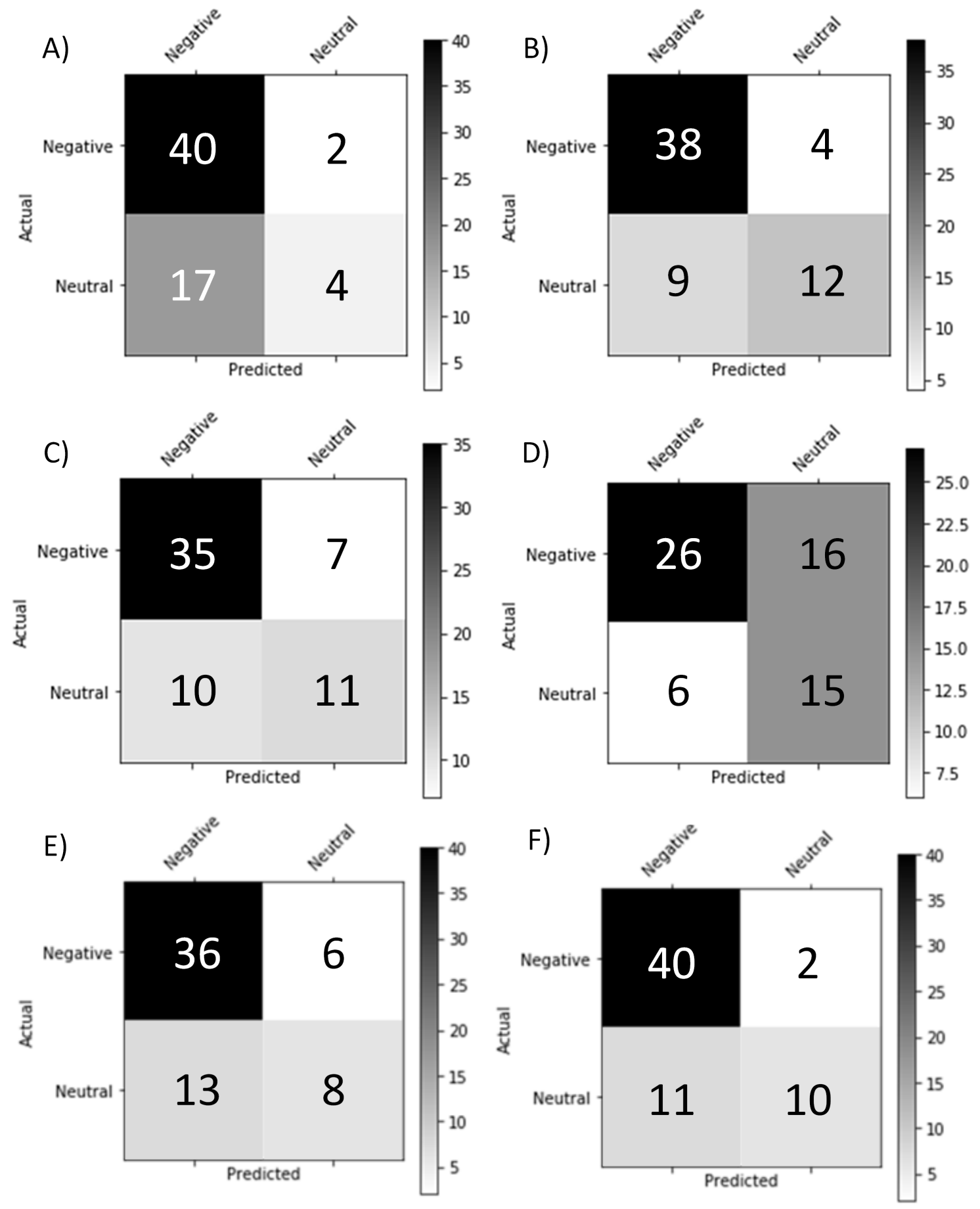

Figure 3.7: A comparison of the confusion matrices obtained from applying the A) Naïve Bayes, B) Logistic Regression C) Support Vector Machine D) Doc2Vec E) Decision Tree F) Random Forest classifiers on the thermal survey responses. 
The six different binary classification algorithms used in this study were naïve Bayes, Logistic Regression, Support Vector Machines, Doc2Vec, Decision Tree and Random Forest. The accuracy of the classification results from each algorithm is shown using a confusion matrix in Figure 3.7. Darker squares on the confusion matrix indicate a higher proportion of true negatives / neutrals while lighter squares demonstrate a higher proportion of false negatives / neutrals. It should be noted that the lowest accuracy achieved through the use of binary classification algorithms resulted in superior results compared to those obtained from the lexicon-based classification approaches. Doc2Vec was found to have the lowest overall percentage accuracy in classifying the tenant sentiments but still achieved a $67 \%$ accuracy compared to $45 \%$ for Vader. Despite Doc2Vec having a lower classification accuracy compared to the rest of the studied classifiers, it was able to correctly classify a superior fraction of the neutral comments compared to the other tested classifiers. The naïve Bayes classifier had the highest correctly predicted fraction of true negatives. However, it fared worse than every other algorithm that was tested while attempting to classify neutral comments. This is possibly due to the dataset having a large bias towards negative comments. Logistic Regression and Random Forest Classifiers were observed to perform the best with this dataset with both achieving an $80 \%$ classification rate. Logistic Regression was found to be more effective while identifying true neutrals while Random Forest Classifiers were found to be more effective at classifying true negatives. Decision Tree Classifiers were unable to match the performance of Random Forest Classifiers with correct classification of both true positives and neutrals. 
Table 3.6: Classifiers used and their percent classification accuracy (for negatives and neutrals combined).

\begin{tabular}{|l|l|}
\hline Classifier Type & Accuracy (\%) \\
\hline Naïve Bayes & 70 \\
\hline Logistic Regression & 79 \\
\hline Support Vector Machine & 73 \\
\hline Doc2Vec (skip-gram) & 67 \\
\hline Decision Tree & 76 \\
\hline Random Forest & 79 \\
\hline
\end{tabular}

\subsection{Discussion and conclusions}

A combination of data visualization, text mining and natural language processing techniques were successfully employed in this study in order to extract operational performance metrics from text-based survey data. Comparisons between survey data (solicited data source) and CMMS data (unsolicited data source) were made; and it was observed that floor-level breakdown of thermal and maintenance complaints were overall similar.

Text mining algorithms were also used on the survey data. The association rule mining method revealed common tenant comments regarding air quality, heating on Mondays, thermostat control in the offices, need for having increased control over floor heating in the winters, issues regarding washroom faucets, soap dispensers, and parking. The topic modelling algorithms reinforced the findings from the association rule mining method by demonstrating distinct topic groups for complaints regarding heating on Mondays, better air circulation, empty soap dispensers, parking and cold summer temperatures. Furthermore, these algorithms allowed for more detailed interpretation of the complaints contained in the dataset including the tenants' desire for access to staircases, high frequency of elevator breakdowns, a desire for better food options within the building and issues regarding revolving doors on the ground 
floor. Topic modelling was found useful for gaining insights into text-based tenant responses.

Sentiment analysis techniques were used in order to quantify overall tenant satisfaction in the survey responses. The survey results were manually classified as negative or neutral as a check for the accuracy of each sentiment analysis algorithm. Lexiconbased algorithms were found to have the worst accuracy in the study. It is possible that these methods are less effective when applied to domain specific datasets (HVAC / maintenance). The development of domain specific dictionaries may have the potential to create gains in accuracy for these algorithms. Classifier-based algorithms had a better accuracy but require a split in the dataset in order to train the algorithm, and a time-consuming manual training process. However, the benefit is that they allow for more accurate models to be developed; training a random forest or logistic regression classifiers allowed for a sentiment analysis accuracy of up to $80 \%$ compared to $45 \%$ for the lexicon-based methods.

The methodology and visualizations obtained through this study have not been used by the industry partner as of yet. However, the results from this analysis should be useful in their operational and maintenance workflows. Based on the results of this study, the primary recommendations with respect to operations changes in order to please tenants would be increased attention on the following issues: a) an availability of soap and running water in washrooms, b) an increase in the scheduled maintenance of the elevators and c) a greater amount of control given to occupants regarding their floor temperatures. This could be in the form of an online tool where they would be able to make thermal changes or an accessible thermostat on the floor whereas currently the thermostats are only accessible by building technicians. The study also points out the three floors with the highest frequency of survey responses and 
demonstrated that the floors which seemed to log more unsolicited feedback (CMMS complaints) and those more likely to respond when solicited for information (tenant surveys). Each of these analytical insights is obtained through the methodology proposed in this study and show the capability of extracting actionable insights from these traditionally overlooked data sources.

The study concludes that free text data contains valuable insights that can translate to improvements in workflow for operators, reduction in tenant complaints which can lead to improvements in their performance indicators and valuable analytics to support data gathered from a word of mouth basis through operators. Despite the difficulty in getting insights from this data source and the push to use various proxies for tenant comfort (IoT devices like smartwatch interactions, CMMS databases, and thermostat interactions), there remains value to using direct text-based feedback from tenants in the analysis of comfort metrics. Techniques which allow for effective text analytics and natural language processing already exist within standard Python libraries and are easily accessible to researchers. The following points were recognized as unresolved issues and are opportunities for future work regarding the topic:

- A greater resolution for performance indicators can be achieved by using room level data to calculate tenant level performance. This data is currently available within the dataset, but it is critical to have a strong understanding of tenant space occupancy as certain tenants within the building occupy multiple floors and rooms across floors.

- A larger dataset needs to be used to show the robustness of these methods. The current dataset has only 431 entries and is very skewed towards negative responses which caused some algorithms like naïve Bayes to have a large bias towards one class during classification. 
- Methods must be developed to lower the rate of misclassification for the neutral comments. Currently even the best algorithm for this purpose (Doc2Vec) only gives us a $71 \%$ accuracy in this parameter.

- Improvements in the accuracy of the sentiment analysis process can be gained by using deep neural networks like RNNs for classification. The popularity of high level interfaces interacting with deep neural network libraries such as the Keras API used with TensorFlow, may allow for more accessibility to these techniques and improve classification accuracy. Early experimentation using a Recurrent Neural Network (RNN) shows very promising results which could indicate that the rate of identification of neutral comments is greatly improved.

- Follow-up interviews with the survey participants were not conducted to verify the results of this study. More collaboration is required as well as a follow up study to demonstrate that building operators can use these survey analytics techniques to help them address tenant complaints. 


\section{Chapter 4}

\section{Development of a graphical interface tool}

\subsection{Introduction}

Tenant complaints from work-order data and operator log entries in CMMS represent an untapped potential to extract detailed performance metrics for building managers and operators. Despite data mining techniques becoming more accessible to researchers through the popularity of languages such as $\mathrm{R}$, non-technical groups (building operators and managers) with an interest in building performance benchmarking must often depend on third parties such as researchers to gain insights into the data generated from their buildings. There remains a gap in the current building management and maintenance field where powerful analytics tools are supplied to building operators and managers who can then visualize the provided building performance metrics at any time.

Research in the field of CMMS analytics has shown that impactful insights can be drawn from both tenant work-orders and operator log entries. Dutta et al., employed association rule mining (ARM) on CMMS data from four buildings to identify building, system, and floor-level work-order taxonomies and common failures [34]. Gunay et al., also used the ARM method to calculate component level fault-frequency rates [8]. Besiktepe et al., analyzed historical work-order data in order to examine 
if there were insightful trends in the types of maintenance activities carried out in educational institutions [35]. However, all these studies require an analyst to be comfortable in programming an analytics workflow in a programming language. It remains to be seen if the powerful analytical techniques used in these studies can be made available to a third party with little or no background on programming and data mining using an intuitive graphical user interface (GUI).

The key challenge regarding development of custom analytical applications for users in an office environment remains the limitations inherent within the corporate structures. Building managers and operators usually work on company owned computers which have carefully moderated software installed on them. This is necessary for offices due to the inherent security threats that are present with running unauthorized and unknown programs on computers. Due to the large volume of potentially sensitive data that may be present in each computer system, IT departments often consider unauthorized programs to be an unacceptable risk. It is impractical for researchers to implement experimental and in-development programs in offices as every change made to the software would have to be vetted by the IT department and cost them working hours. In order to avoid this issue, analytics programs can be written for these offices using pre-approved software such as Visual Basic for Applications (VBA) for Microsoft Excel, but the opportunity cost associated with taking this approach is the current availability of mature, robust and well established libraries for data and text analytics within the $\mathrm{R}$ ecosystem.

In this article, a prototype software tool to extract operational performance metrics from work-order logs of large commercial and institutional buildings is developed with established text and data analytics techniques running on the back end and an intuitive GUI on the front end. The goal of this tool is to create an interface 
where non-technical building staff can have access to complex yet powerful data analytics techniques like survival curves, stacked line curves, Sankey diagrams and association node networks in order to draw insights on building performance and work-order taxonomies without having to refer to a researcher or specialist in data analytics. The shortcomings of the methodology are identified through interactions with operations staff; and future research needs are discussed regarding operational data collection, analytics, and visualisation.

\subsection{Methods}

The source code for the performance metrics used in this project was obtained from the open source documentation provided by Dutta et al [43]. The $\mathrm{R}$ language for statistical computing was utilized for the development of this software tool. $\mathrm{R}$ is an open source language with powerful third-party packages which allows data analysts to clean, process, analyze and visualize data. $\mathrm{R}$ contains the package shiny which allows for the creation of applications with a GUI using code originally written for statistical analysis. Non-technical users can take advantage of an intuitive GUI to obtain analytical insights using data and text mining techniques built into the back end of the application without any prior knowledge of programming. The second advantageous feature of shiny applications are their ability to run on cloud instances. Instead of having the application installed on a number of local computers, building managers can simply choose to have the application run on a hosting server. Employees interested in analyzing the building data can then simply access the application through their browser by connecting to the server. Benchmarking data will be uploaded to the server in the form of a comma-separated values file that building staff 
can access from their CMMS system.

The advantages of a cloud-based approach to an application are numerous. The primary advantage is the ease of version control and bug testing; developers need to update the application on one server only rather than pushing updates to multiple local computers. Any bugs within the application are also easier to find and patch due to the application running on a single known system. It also eliminates the need for developers to consider the hardware capabilities of each user as the computing power required is managed by the cloud instance. Finally, recall that one of the challenges for deploying in development tools for offices is the need for IT departments to validate individual programs as secure before they are deployed on a local system. The use of a cloud-based application which uses an uploaded file for benchmarking allows for this challenge to be bypassed. Researchers can create updates to the application and have them provided to building staff much faster without having to go through another level of verification in between. The five building parameters that were analyzed in the prototype tool were the "per category work-order resolution time", "per category work-order intensity", "work-order per floor distribution", "top ten operator word frequency" and "operator term usage patterns". The techniques used to derive these benchmarks and their significance to building operations are discussed below. 


\subsubsection{Per category work-order resolution time}

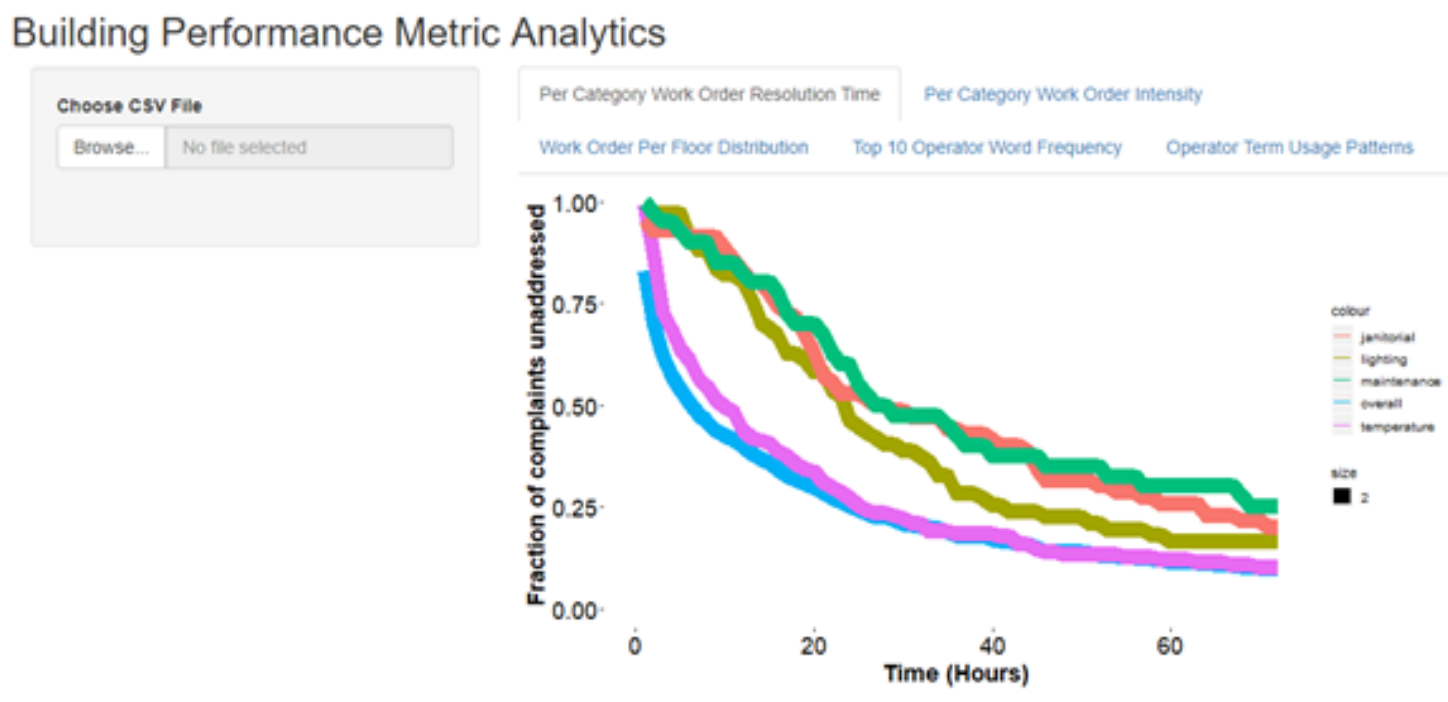

Figure 4.1: Work-order resolution time benchmarking using survival curves.

Survival curves are used in order to demonstrate the performance of a building with respect to how quickly work-orders are addressed. For example, the janitorial, lighting and maintenance work-orders in the building in Figure 4.1 took longer to address compared to the temperature-related work-orders. Within the studied dataset, only $30 \%$ of temperature-related work-orders were unaddressed at the 20-hour mark whereas $75 \%$ of janitorial and maintenance and $56 \%$ of lighting work-orders remained unresolved. At the 40-hour mark, $28 \%$ of lighting-related work-orders remained unresolved while $43 \%$ and $48 \%$ of janitorial- and maintenance-related work-orders were unaddressed, respectively. In this scenario, a conclusion the building managers could draw is that there is an opportunity for the building staff to improve on their response times regarding janitorial and maintenance work-orders. 


\subsubsection{Per category work-order intensity}

\section{Building Performance Metric Analytics}

Choose CsV File

Browse... No the selectied

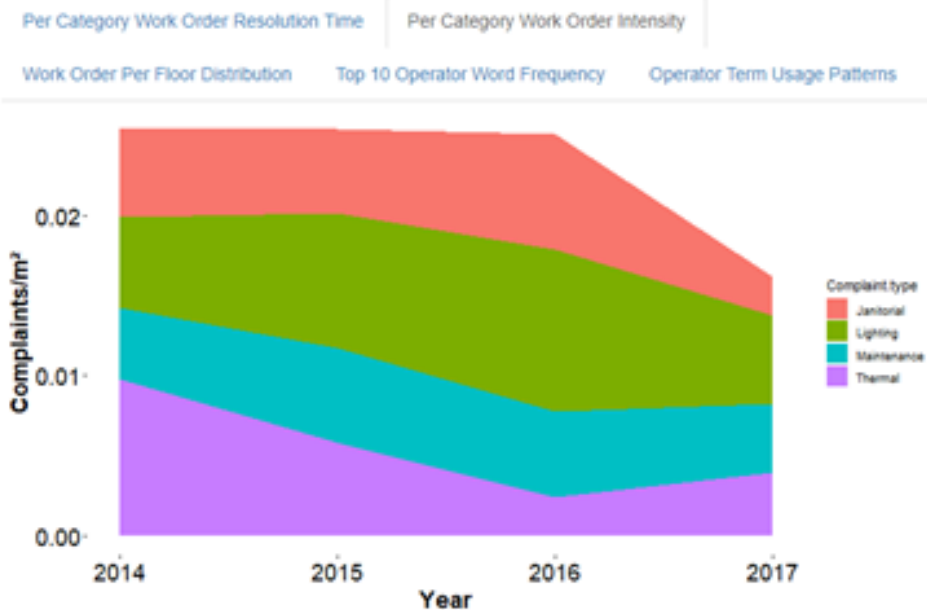

Figure 4.2: Complaint intensity normalized by floor area using service request counts.

Stacked line charts are useful for demonstrating the change in complaint intensity normalized by floor area of a building. As shown in Figure 4.2, interesting observations for the building include an overall decrease in temperature and janitorial work-orders per square meter by $64 \%$ and $57 \%$ between 2014 and 2017, respectively. This is a strong indication that the facilities management team were successful in improving their workflows regarding thermal work-orders and janitorial work-orders significantly between 2014 and 2017. In contrast to the reduction in complaint intensity witnessed with the previous categories, lighting and maintenance work-orders saw no reduction in complaint intensity and hence there may be an opportunity for improving performance regarding those types of work-orders. 


\subsubsection{Work-order per floor distribution}

\section{Building Performance Metric Analytics}

Choore CsV File

Browse... No the selected

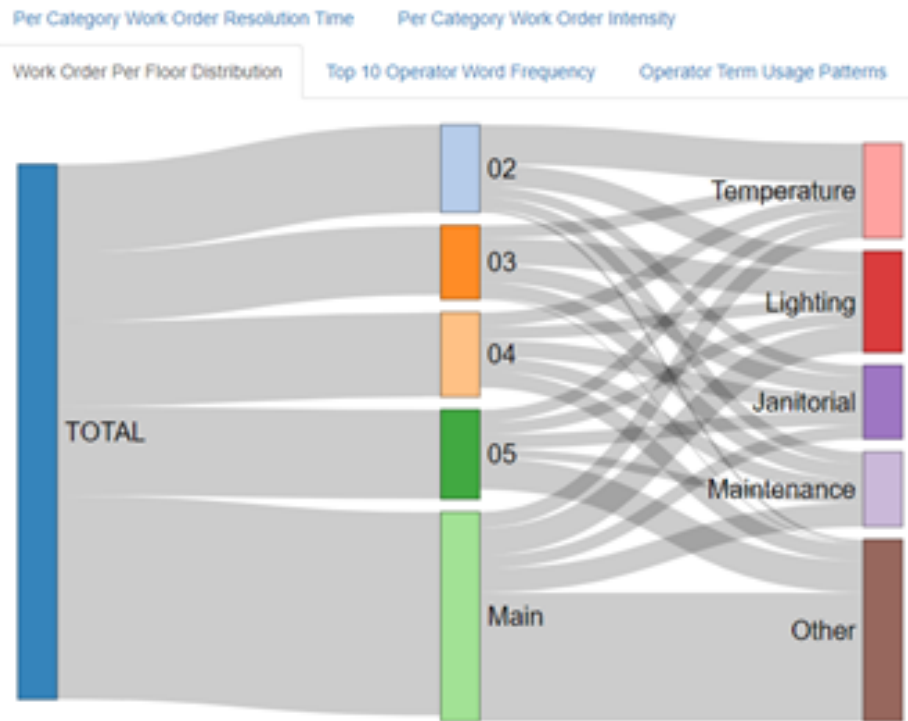

Figure 4.3: A per floor and per type breakdown of tenant service requests.

Sankey diagrams provide insight into categorical and spatial breakdown of the work-orders within a building. It is often observed that only a few areas of a building (i.e. floors, rooms) are associated with disproportionate numbers of work-orders which are often of a certain type (thermal, lighting etc.). Presenting a per floor and a per category breakdown of the work-orders provides insight into areas where disproportionate numbers of work-orders are generated. This is an efficient way of increasing tenant satisfaction by efficiently identifying and addressing problems at a high resolution. For example, the category of work-orders from which the highest volume of work-orders was observed on the third floor was lighting. Additionally, a disproportionate number of work-orders regarding temperature were observed on the second floor. This data would ideally be used by a building manager to start a more detailed investigation into any underlying causes that may have led to the generation 
of a disproportionate number of work-orders.

\subsubsection{Top 10 operator word frequency}

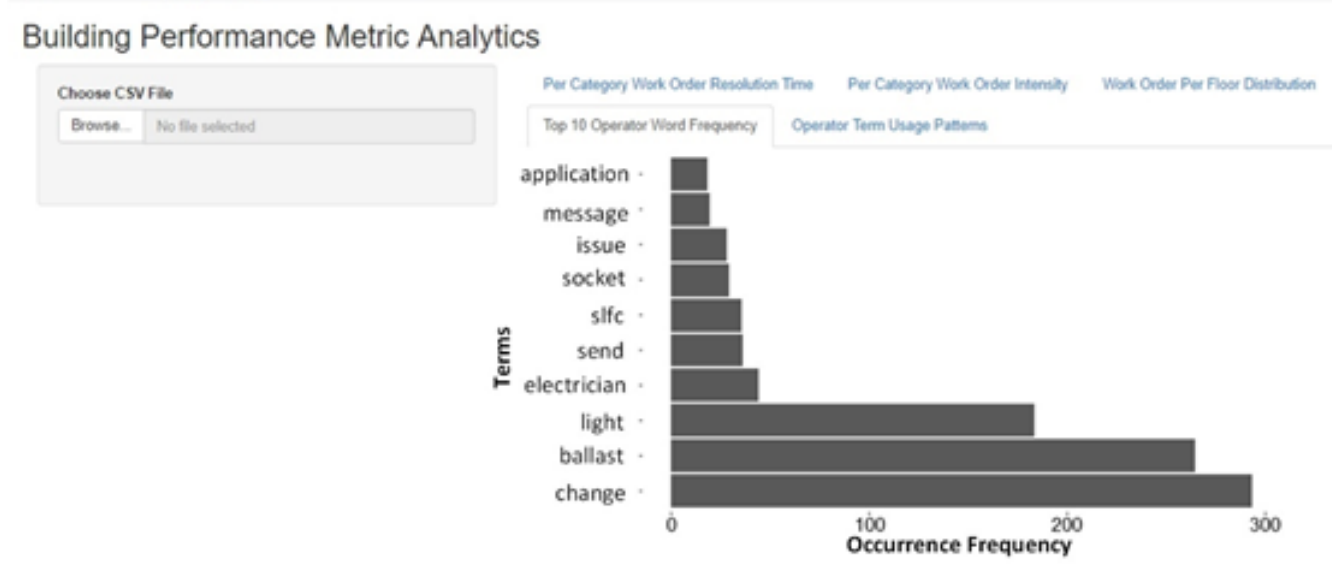

Figure 4.4: Word frequencies of operator log entries for lighting related service requests.

Frequently occurring terms within the operator logs in the CMMS database can help building managers pinpoint key trends/problems that operators face as they resolve complaints. Bar graphs were used to demonstrate the top ten words used by operators while responding to work-orders. Notably, the most frequent words the building operators use while addressing maintenance complaints categories are replace, tenant, satisfy and material. 


\subsubsection{Operator term usage patterns}

\section{Building Performance Metric Analytics}

Choose CsV File

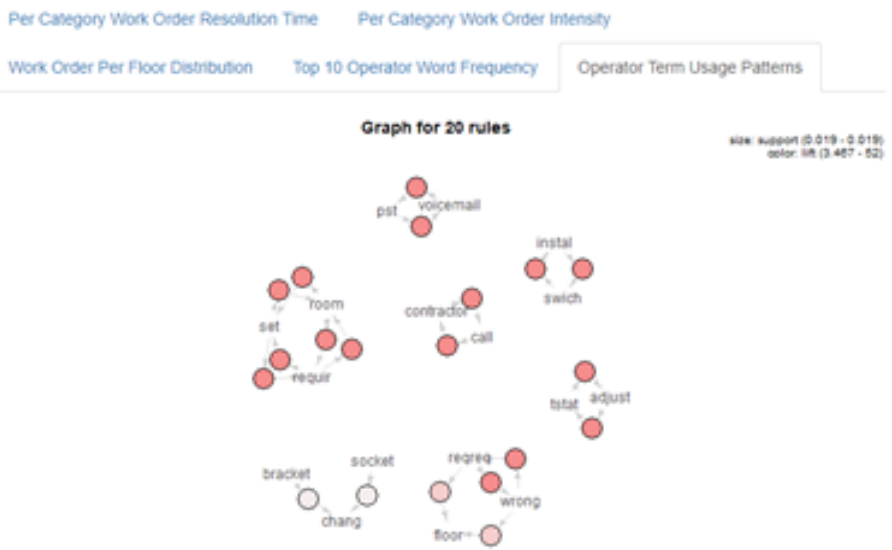

Figure 4.5: Word frequencies of operator log entries for lighting related service requests.

Association node networks allow for the easy interpretation of the top rules identified by the ARM technique which identifies frequently co-occurring words within a dataset. For example, if the word "tstat" appears frequently alongside the word "change" in the dataset, the inference is that operators frequently are called up to readjust temperatures in spaces.

Larger nodes indicate a greater term frequency within the analyzed dataset while darker nodes signify terms with a greater probability of occurring together. Figure 4.5 shows a graphical representation of the rules that were discovered when exploring the irregularities found in maintenance-related complaints on the building work-order entries. It is evident that looking at the maintenance-related complaints in this building that operators are frequently installing switches and adjusting the thermostats. 


\subsection{Future performance metrics and visualization}

Additional performance metrics are planned as additions to the ones already discussed. Since these metrics have not been implemented in the application as of this point, a non-functional graphical user interface has been developed with the proposed functionality of the performance metrics integrated within. The non-functional interface is a representation of what the finalized product is envisioned to resemble.

\subsubsection{Temporal distribution of complaints}

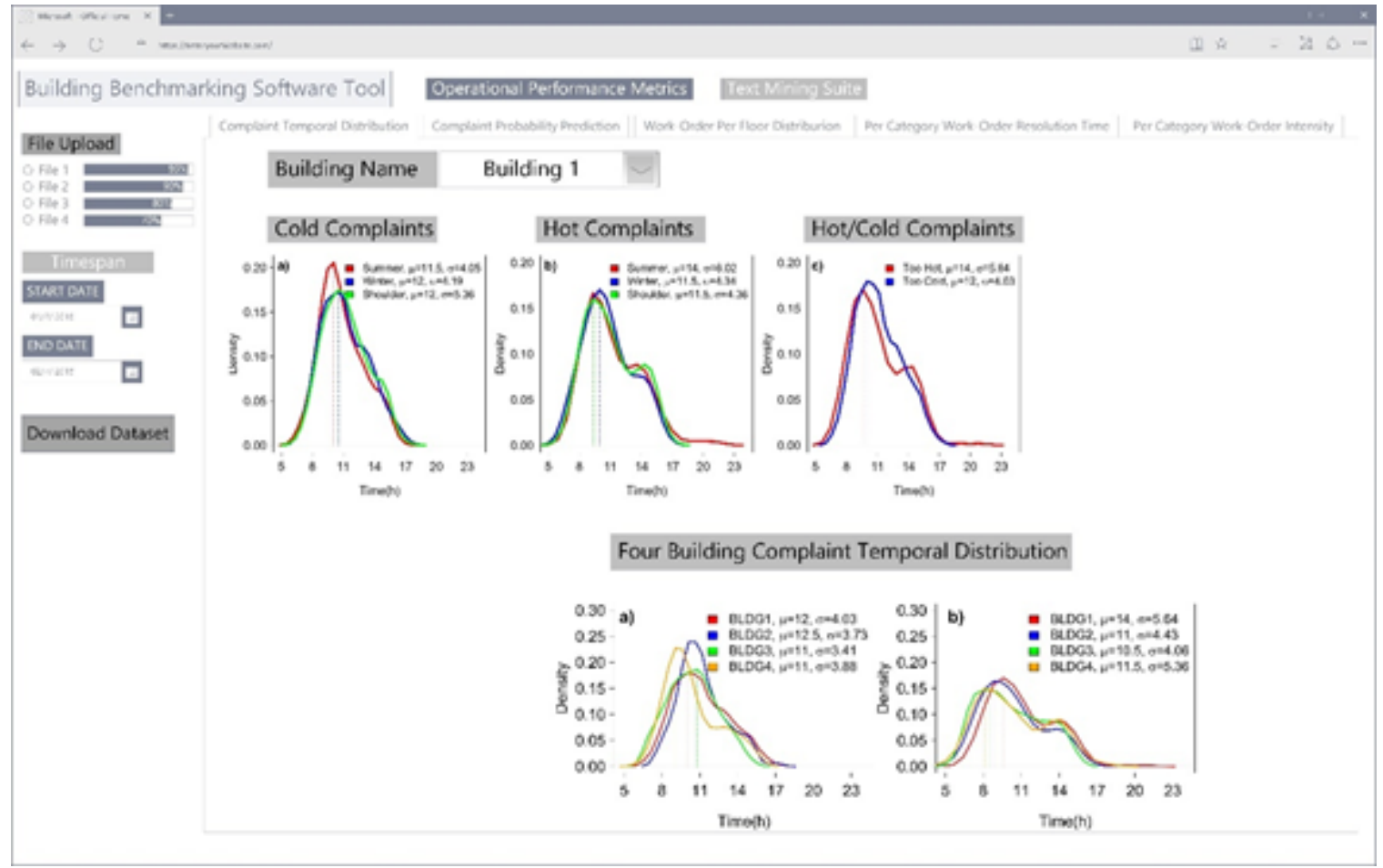

Figure 4.6: Temporal distributions of complaints over a 24-hour period.

Temporal distributions of complaints throughout a workday (24 hours) are visualized using probability density functions for up to four uploaded buildings. Hot and cold complaints are separated as categories in order to differentiate between the 
trends they show. Temporal differences in the complaints are displayed using three bins to break down the complaint trends for the summer, winter and shoulder seasons as shown in Figure 4.6. The detailed temporal complaint distributions can be studied from building to building by selecting one of the uploaded building datasets. A comparison of the temporal complaint distribution between the four uploaded buildings is also provided. Of interest to building managers from this visualization would be the peaks in the density functions; these would correspond with the peak complaint times. For the example dataset, this appeared to be around 10:00 AM. The shape of the complaint probability function also alerts managers to irregular patterns which may signal operations issues. In the example dataset, several buildings demonstrated secondary peaks for hot complaints at around 2:00 PM during the workday. This is a possible indication of solar heat gains causing an overheating issue in the space later on during the workday. 


\subsubsection{Average 12 hour complaint trees}

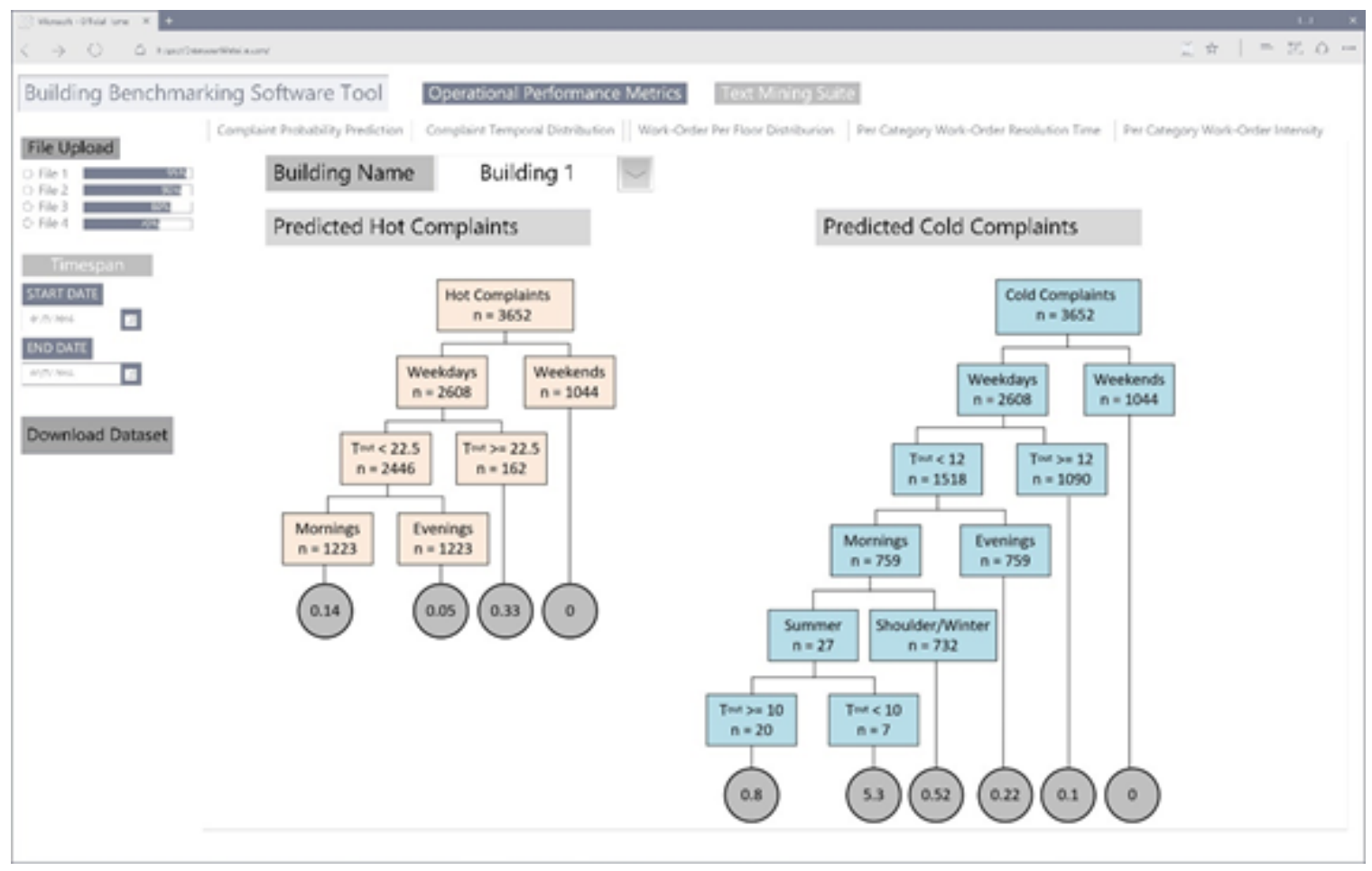

Figure 4.7: Decision trees demonstrating the effect of outdoor temporal and spatial factors on average complaint rates over a $12 \mathrm{~h}$ period.

Decision trees present the effects of temporospatial factors on the number of hot and cold complaints that are logged in a day. The decision trees shown in Figure 4.7 were constructed using CMMS complaint data from a sample building. The two decision tree models were developed for hot and cold complaints using four years of data collected from complaint logs. The variable $\mathrm{n}$ within each tree refers to the number of 12-hour timesteps in a bin within the dataset. The decision tree consists of nodes (squares) and leaves (circles). The nodes of the tree quantify statistically significant splits within the dataset while the leaves predict the number of complaints that would occur within a 12-hour timespan. This allows building operators and managers to statistically predict the conditions under which complaints would be 
maximized. As an example, managers for the studied building could expect the maximum rate of cold complaints during summer when the outdoor temperature was less than 10 degrees Celsius outdoors. This could indicate an AHU schedule which runs on a fixed schedule and turns on the cooling regardless of the outdoor temperature during the summer season. Creating better HVAC schedules in this case would serve to both save the building managers money and increase tenant satisfaction.

\subsection{Future application features and development goals}

The application as is presented in this chapter is still at a stage of ongoing development. Future plans for the application include:

1. Compare performance metrics between up to four buildings normalized by floor area;

2. Custom user defined time periods for building performance analysis;

3. Choice of the complaint categories (e.g., thermal, lighting, maintenance etc.) for the word frequency charts of operator log entries;

4. User control of the number of top words included within the charts;

5. Association node network graphs based on user preference of support, lift and confidence for the apriori algorithm and the ability to vary the values of those parameters as required; 


\subsection{Feedback and concluding remarks}

Preliminary feedback from building managers, building operators and developers indicates a desire to have increased control over the application than is granted to the user in its present state. The building managers noted that a vital part of their workflow regarding the performance metrics they obtain from the application would be to compare them between buildings. For example, there was a strong demand for having the ability to compare work-order resolution benchmarks between buildings and compare components of that benchmark (e.g., lighting or thermal work-order resolution performance) between buildings under the same management team. There is also a request for greater documentation of the performance metrics explaining what each metric represents and how building management factors could tie into the trends operators and managers observe from the application.

The application demonstrates a proof of concept that insights derived from analytical tools requiring knowledge of complex programming languages can be brought to non-technical users through a GUI. The application allows building operators and managers to obtain continuous on-site, real time performance metrics as opposed to sending data to third parties for annual/bi-annual analysis. Additionally, nontechnical building staff can make use of the application without having any prior programming knowledge and deploy it on their system without having to certify it with their IT departments. More work needs to be done on the usability of the app lication and the metrics it presents. It is also imperative to add in features that allow the user more control such as the ability to compare metrics from multiple buildings and the ability to infer insights from multiple types of complaints (thermal, lighting etc.). Future development of this application will address the concerns that have been raised as it is aimed at being an ongoing project. 


\section{Chapter 5}

\section{Conclusions}

\subsection{Summary}

Innovative data mining, analytical and visualization techniques were employed in this research in order to develop performance metrics for buildings using CMMS and free text survey datasets. A summary of the conclusions from Chapters 2-4 of this research is outlined as follows.

\subsubsection{Extracting performance metrics with work-order data}

Chapter 2 demonstrated the potential of complaint logs and operator comments stored within computerized maintenance management systems in developing operational performance metrics for buildings. The methodology for analysis was to break complaints down (per floor, per category, per building etc.) in order to explore temporospatial trends and create building level benchmarks that are intuitive to understand for operators. The major conclusions derived from this research are:

- only certain complaint categories contribute to a majority of the recorded complaints.

- all four buildings experienced a peak complaint frequency for both hot and cold 
complaints around $12 \mathrm{pm}$.

- hot and cold complaints had distinctive frequency distributions, with hot complaints featuring a prominent secondary peak in complaint intensity.

- analysis showed that the highest probability of thermal and air quality complaints occurred on only a very small number of days during the shoulder season.

- text mining operator work-orders reveals key operational procedures which are repeated several times, highlighting trends in operational and maintenance workflows.

\subsubsection{Insight extraction from tenant surveys using text min- ing}

In Chapter 3, well established data visualization, text mining and natural language processing algorithms were used in order to extract operational performance metrics from text-based survey data. A methodology was developed in order to automate the process of insight extraction from text based data and the effectiveness of several well established algorithms in providing interpretable results was evaluated. Sankey diagrams were used to compare the complaint patterns between solicited (surveys) and unsolicited (CMMS) complaint sources, association rule mining was used to establish trends within the terms used in tenant complaints, topic modelling was employed in order to isolate groups of issues that tenants frequently mentioned and the feasibility of multiple, well established sentiment analysis methods were assessed in order to predict their effectiveness in establishing tenant satisfaction. The major conclusions 
derived from this research were:

- floor-level breakdown of thermal and maintenance complaints were similar when comparing solicited (surveys) and unsolicited (CMMS) complaint data.

- the association rule mining method revealed common tenant comments regarding air quality, thermostat control in the offices, issues regarding washroom faucets and soap dispensers.

- the results from the topic modelling algorithms reinforced the results found through association rule mining.

- topic modelling allowed for more detailed interpretation of the complaints contained in the dataset including desire for access to staircases, high frequency of elevator breakdowns and issues regarding revolving doors on the ground floor.

- lexicon-based algorithms with a generic dictionary were found to have the worst accuracy among the studied methods. It is possible that these methods are less effective when applied to domain specific datasets (HVAC / maintenance).

- classifier based sentiment analysis algorithms allow for more accurate models to be developed at the cost of more time involvement in the training process.

- results from insight extraction using multiple methods (ARM and topic modelling) converged into similar insights.

\subsubsection{A software tool for conducting data analytics}

In Chapter 4, a functional application is developed with a graphical user interface. The performance metrics which are developed from Chapters 2 and 3 of the study 
are integrated within the back end of the application. An intuitive front end is developed in order to allow building managers an opportunity to obtain on demand performance data from the developed benchmarking tools. The application is built to be deployable on a cloud platform for optimal scalability and development efficiency. A roadmap for future development is laid out.

\subsubsection{Envisioned commercial usage}

The work as presented in this thesis is envisioned to be deployed in a commercial setting under a facilities management company with several buildings under their portfolio. Ideally these buildings would have reserves of CMMS and tenant survey data which they would like to draw insights from. A commercial deployment of this study would be done through the developed application (either locally or the cloud) and building operators and managers would be able to upload data they want analyzed through a CSV file. Depending on the data type they would then be presented with a visual benchmark suite that consists of survival curves, sankey diagrams, decision trees, association node networks, topic modeling insight etc. Furthermore, the tool could be directly automated with the servers storing the CMMS and survey data to provide automatic monthly/weekly reports containing detailed insights into operations/faults which would otherwise be very difficult to detect using simple energy use analysis. 


\subsubsection{Contributions}

The following are the research contributions of this work:

- Developed a methodology for developing performance metrics from tenant complaint entries and operator service logs in CMMS.

- Created operational performance metrics and text mining workflows for detailed insight extraction from tenant survey datasets.

- Developed a prototype software application and open source library of functions for future work on CMMS systems and free text survey datasets.

\subsection{Recommendations for future work}

This section discusses the overall recommendations for future work as it pertains to the body of research within this thesis. The three recommended segments of future work are improvements on the text analytics methodology, future application development and commercial deployment of the application.

\subsubsection{Improvements on the text analytics methodology}

Although novel approaches were used in order to extract performance insight from text based survey datasets, there are several steps that can be undertaken to further refine the methodology.

- larger datasets located across differnt climate zones must be evaluated in order to test the performance of the workflow on more data, and data that is not as skewed in distribution as the one used in this study. 
- further research must be undertaken to improve the classification of survey responses with a neutral polarity.

- research must be conducted into the application of deep neural networks (CNNs) for text classification.

- lexicon based sentiment analysis techniques must be reassessed using domain specific dictionaries to improve classification performance.

- the accuracy of the developed classifier models must be tested on tenant survey datasets from other buildings to test their transferability.

- better structured text based survey formats can be designed such that tenant responses have less variance and focus on a few key issues of interest to building operators.

\subsubsection{Roadmap for application development}

The custom building benchmarking application is still in its infancy. A few initial features have been implemented within it as a prototype. However, a number of features must be added to it so that a robust set of benchmarking tools are available to building managers. The application must also be tested with multiple databases to account for the differences in storage formats of computerized maintenance management systems. Some of the future development goals towards the application are listed below:

- user control of temporal factors within the benchmarks.

- comparison of multiple buildings for a performance metric. 
- integration of text mining algorithms (topic modelling, classification etc.) into the application.

- application bug testing and performance metric validation.

- initial feedback about the GUI and operational metrics from building managers and operators.

- deployment on the cloud for industry use and continued development based on feedback. 


\section{List of References}

[1] L. Pérez-Lombard, J. Ortiz, R. González, and I. R. Maestre, "A review of benchmarking, rating and labelling concepts within the framework of building energy certification schemes," Energy and Buildings, vol. 41, no. 3, pp. 272-278, 2009.

[2] W. Kim and S. Katipamula, "A review of fault detection and diagnostics methods for building systems," Science and Technology for the Built Environment, vol. 24, no. 1, pp. 3-21, 2018.

[3] M. Deru and P. Torcellini, "Performance Metrics Research Project - Final Report," no. October, 2005.

[4] T. Hong, L. Yang, D. Hill, and W. Feng, "Data and analytics to inform energy retrofit of high performance buildings," Applied Energy, vol. 126, pp. 90-106, 2014.

[5] M. T. Paulus, D. E. Claridge, and C. Culp, "Algorithm for automating the selection of a temperature dependent change point model," Energy and Buildings, vol. 87, no. November, pp. 95-104, 2015.

[6] Z. Li, Y. Han, and P. Xu, "Methods for benchmarking building energy consumption against its past or intended performance: An overview," Applied Energy, vol. 124, pp. 325-334, 2014.

[7] Z. Wang and R. S. Srinivasan, "A review of artificial intelligence based building energy use prediction: Contrasting the capabilities of single and ensemble prediction models," Renewable and Sustainable Energy Reviews, vol. 75, pp. 796-808, 2017.

[8] H. B. Gunay, W. Shen, and C. Yang, "Text-mining building maintenance work orders for component fault frequency," Building Research and Information, vol. 47, pp. 518-533, 2019.

[9] Y. Geng, W. Ji, Z. Wang, B. Lin, and Y. Zhu, "A review of operating performance in green buildings: Energy use, indoor environmental quality and occupant satisfaction," Energy and Buildings, vol. 183, pp. 500-514, 2019. 
[10] K. Y. Teichman, A. K. Persily, and S. J. Emmerich, "Indoor air quality in highperforming building case studies: Got data?," Science and Technology for the Built Environment, vol. 21, no. 1, pp. 91-98, 2015.

[11] K. W. Tham, P. Wargocki, and Y. F. Tan, "Indoor environmental quality, occupant perception, prevalence of sick building syndrome symptoms, and sick leave in a Green Mark Platinum-rated versus a non-Green Mark-rated building: A case study," Science and Technology for the Built Environment, vol. 21, no. 1, pp. 35-44, 2015.

[12] G. R. Newsham, B. J. Birt, C. Arsenault, A. J. Thompson, J. A. Veitch, S. Mancini, A. D. Galasiu, B. N. Gover, I. A. MacDonald, and G. J. Burns, "Do green buildings have better indoor environments? New evidence," Building Research and Information, vol. 41, no. 4, pp. 415-434, 2013.

[13] G. R. Newsham, J. A. Veitch, and Y. V. Hu, "Effect of green building certification on organizational productivity metrics," Building Research and Information, vol. 46, no. 7, pp. 755-766, 2018.

[14] T. Sood, M. Quintana, P. Jayathissa, M. Abdelrahman, and C. Miller, "The SDE4 Learning Trail: Crowdsourcing occupant comfort feedback at a net-zero energy building," Journal of Physics: Conference Series, vol. 1343, no. 1, 2019.

[15] H. B. Gunay, W. Shen, G. Newsham, and A. Ashouri, "Modelling and analysis of unsolicited temperature setpoint change requests in office buildings," Building and Environment, 2018.

[16] W. O’Brien, I. Gaetani, S. Carlucci, P. J. Hoes, and J. L. Hensen, "On occupantcentric building performance metrics," Building and Environment, vol. 122, pp. 373-385, 2017.

[17] M. M. Ouf, W. O’Brien, and B. Gunay, "On quantifying building performance adaptability to variable occupancy," Building and Environment, vol. 155, no. February, pp. 257-267, 2019.

[18] D. Hammond, J. J. Dempsey, F. Szigeti, and G. Davis, "Integrating a performance-based approach into practice: A case study," Building Research and Information, vol. 33, no. 2, pp. 128-141, 2005.

[19] J. Goins and M. Moezzi, "Linking occupant complaints to building performance," in Building Research and Information,vol. 42, no. 3, pp. 362-372, 2013. 
[20] M. Moezzi and J. Goins, "Text mining for occupant perspectives on the physical workplace," Building Research and Information, vol. 39, no. 2, pp. 169-182, 2011.

[21] J. J. McArthur, N. Shahbazi, R. Fok, C. Raghubar, B. Bortoluzzi, and A. An, "Machine learning and BIM visualization for maintenance issue classification and enhanced data collection," Advanced Engineering Informatics, vol. 38, no. October 2017, pp. 101-112, 2018.

[22] C. C. Federspiel, "Predicting the frequency and cost of hot and cold complaints in buildings," HVACER Research, vol. 6, no. 1, pp. 289-305, 2001.

[23] D. Wang, C. C. Federspiel, and E. Arens, "Correlation between temperature satisfaction and unsolicited complaint rates in commercial buildings," Indoor Air, vol. 15, no. 1, pp. 13-18, 2005.

[24] R. Bortolini and N. Forcada, "Analysis of building maintenance requests using a text mining approach: building services evaluation," Building Research and Information, vol. 48, no. 2, pp. 207-217, 2019.

[25] B. Bordass, R. Cohen, M. Standeven, and A. Leaman, "Assessing building performance in use 2: technical performance of the probe buildings," Building Research E Information, vol. 29, no. 2, pp. 103-113, 2001.

[26] H. Kato, L. Too, and A. Rask, "Occupier perceptions of green workplace environment: The Australian experience," Journal of Corporate Real Estate, vol. 11, no. 3, pp. 183-195, 2009.

[27] A. Keyvanfar, A. Shafaghat, M. Z. A. Majid, H. Lamit, and K. N. Ali, "Correlation study on user satisfaction from adaptive behavior and energy consumption in office buildings," Jurnal Teknologi, vol. 70, no. 7, pp. 89-97, 2014.

[28] "The Well Building Standard," url: https://a.storyblok.com/f/52232/x/406c235c09/wellv1-with-q4-2019-addenda.pdf, 2019.

[29] G. R. Newsham, B. J. Birt, C. Arsenault, A. J. Thompson, J. A. Veitch, S. Mancini, A. D. Galasiu, B. N. Gover, I. A. MacDonald, and G. J. Burns, "Do green buildings have better indoor environments? New evidence," Building Research and Information, vol. 41, no. 4, pp. 415-434,2:01 PM 2020-03-22 2013.

[30] H. Burak Gunay, W. Shen, and G. Newsham, "Data analytics to improve building performance: A critical review," Automation in Construction, vol. 97, no. June 2018, pp. 96-109, 2019. 
[31] C. C. Federspiel, "Predicting the Frequency and Cost of Hot and Cold Complaints in Buildings," in ASHRAE Winter Meeting CD, Technical and Symposium Papers, 2001.

[32] D. Wang, C. C. Federspiel, and E. Arens, "Correlation between temperature satisfaction and unsolicited complaint rates in commercial buildings," Indoor Air, vol. 15, no. 1, pp. 13-18, 2005.

[33] H. B. Gunay, W. Shen, and C. Yang, "Text-mining building maintenance work orders for component fault frequency, vol. 47, no. 5, pp. 518-533, 2018.

[34] S. Dutta, H. B. Gunay, and S. Bucking, "A method for extracting performance metrics using work-order data A method for extracting performance metrics using work- order data," Science and Technology for the Built Environment, vol. 0, no. 0, pp. 1-12, 2019.

[35] D. Besiktepe, M. E. Ozbek, and R. A. Atadero, "Analysis of the maintenance work order data in educational institutions," ISEC 2019 - 10th International Structural Engineering and Construction Conference, no. May, 2019.

[36] A. Leaman and B. Bordass, "Productivity in buildings: the 'killer' variables," Building Research ES Information, vol. 27, no. 1, pp. 4-19, 1999.

[37] S. Karjalainen, "Thermal comfort and use of thermostats in Finnish homes and offices," Building and Environment, vol. 44, no. 6, pp. 1237 - 1245, 2009.

[38] H. B. Gunay, W. O'Brien, I. Beausoleil-Morrison, and J. Bursill, "Development and implementation of a thermostat learning algorithm," Science and Technology for the Built Environment, vol. 24, no. 1, pp. 43-56, 2018.

[39] T. Peffer, M. Pritoni, A. Meier, C. Aragon, and D. Perry, "How people use thermostats in homes: A review," Building and Environment, vol. 46, no. 12, pp. 2529-2541, 2011.

[40] P. Jayathissa, M. Quintana, T. Sood, N. Narzarian, and C. Miller, "Is your clockface cozie ? A smartwatch methodology for the in-situ collection of occupant comfort data," CISBAT2019 Climate Resilient Buildings - Energy Efficiency \& Renewables in the Digital Era, 2019.

[41] J. Goins and M. Moezzi, "Links between occupant complaint handling and building performance, vol. 42, no. 3, pp. 362-372, 2012. 
[42] M. Moezzi and J. Goins, "Text mining for occupant perspectives on the physical workplace," Building Research and Information, vol. 42, no. 3, pp. 362-372, 2011.

[43] S. Dutta, "Comparison of Sentiment Analysis Methods," url: https://github.com/saptakdutta/Sentiment-analysis, 2019.

[44] D. M. Blei, A. Y. Ng, and M. I. Jordan, "Latent Dirichlet allocation," Journal of Machine Learning Research, vol. 3, no. 4-5, pp. 993-1022, 2003.

[45] M. Taboada, J. Brooke, and K. Voll, "Lexicon-Based Methods for Sentiment Analysis," Computational Linguistics, vol. 37(2), pp. 267-307, 2011.

[46] R. Bekkerman, A. Mccallum, and G. Huang, "Automatic categorization of email into folders: Benchmark experiments on Enron and SRI corpora, vol. 418, no. 1, pp. 1-23, 2004.

[47] T. Joachims, "Text categorization with Support Vector Machines: Learning with many relevant features BT - Machine Learning: ECML-98," (Berlin, Heidelberg), pp. 137-142, Springer Berlin Heidelberg, 1998.

[48] J. Li, G. Huang, C. Fan, Z. Sun, and H. Zhu, "Key word extraction for short text via word2vec, doc2vec, and textrank," Turkish Journal of Electrical Engineering and Computer Sciences, vol. 27, no. 3, pp. 1794-1805, 2019. 